\title{
Exponents of Diophantine approximation
}

\author{
Yann Bugeaud
}

We survey classical and recent results on exponents of Diophantine approximation. We give only a few proofs and highlight several open problems.

\section{Introduction and generalities}

Let $\xi$ be an irrational real number. It follows from the theory of continued fractions that there are infinitely many rational numbers $p / q$ with $q \geq 1$ and such that

$$
|q \xi-p| \leq q^{-1}
$$

Said differently, for arbitrarily large integers $Q$, there exist integers $p$ and $q$ with $1 \leq q \leq Q$ and $|q \xi-p| \leq Q^{-1}$. The Dirichlet Theorem asserts much more, namely that, for every integer $Q \geq 1$, there exist integers $p$ and $q$ with $1 \leq q \leq Q$ and $|q \xi-p| \leq Q^{-1}$.

A question arises then naturally: is there some specific irrational real number $\xi$ for which it is possible to improve the above statements, that is, to get the above inequalities with $Q^{-1}$ replaced by $Q^{-w}$ for some real number $w>1$. This leads to the introduction of the exponents of approximation $w_{1}$ and $\hat{w}_{1}$.

Definition 1.1. Let $\xi$ be a real number. We denote by $w_{1}(\xi)$ the supremum of the real numbers $w$ for which there exist arbitrarily large integers $Q$ and integers $p$ and $q$ with $1 \leq q \leq Q$ and

$$
|q \xi-p| \leq Q^{-w}
$$

We denote by $\hat{w}_{1}(\xi)$ the supremum of the real numbers $\hat{w}$ such that, for every sufficiently large integer $Q$, there are integers $p$ and $q$ with $1 \leq q \leq Q$ and

$$
|q \xi-p| \leq Q^{-\hat{w}}
$$

2000 Mathematics Subject Classification : 11J13. To be published in: 'Dynamics and Number Theory', Proceedings of the Durham Easter School 2014. 
As observed by Khintchine [41], every irrational real number $\xi$ satisfies $\hat{w}_{1}(\xi)=1$, since there are arbitrarily large integers $Q$ such that the inequality $|q \xi-p| \leq 1 /(2 Q)$ has no solutions in integers $p, q$ with $1 \leq q \leq Q$; see the proof of Proposition 2.4 below. However, for any $w>1$, there exist irrational real numbers $\xi$ such that, for arbitrarily large integers $Q$, the equation

$$
|q \xi-p| \leq Q^{-w}
$$

has a solution in integers $p$ and $q$ with $1 \leq q \leq Q$. It suffices for instance to take $\xi=\sum_{j \geq 1} 2^{-\left\lfloor(w+1)^{j}\right\rfloor}$, where $\lfloor\cdot\rfloor$ denotes the integer part.

Obviously, the quality of approximation strongly depends upon whether we are interested in a uniform statement (that is, a statement valid for every $Q$, or for every sufficiently large $Q$ ) or in a statement valid only for arbitrarily large values of $Q$.

The general framework is the following. For any (column) vector $\underline{\theta}$ in $\mathbf{R}^{n}$, we denote by $|\underline{\theta}|$ the maximum of the absolute values of its coordinates and by

$$
\|\underline{\theta}\|=\min _{\underline{y} \in \mathbf{Z}^{n}}|\underline{\theta}-\underline{y}|
$$

the maximum of the distances of its coordinates to the rational integers.

Definition 1.2. Let $n$ and $m$ be positive integers and $A$ a real matrix with $n$ rows and $m$ columns. Let $\underline{\theta}$ be an $n$-tuple of real numbers. We denote by $w(A, \underline{\theta})$ the supremum of the real numbers $w$ for which, for arbitrarily large real numbers $X$, the inequalities

$$
\|A \underline{x}-\underline{\theta}\| \leq X^{-w} \quad \text { and } \quad|\underline{x}| \leq X
$$

have a solution $\underline{x}$ in $\mathbf{Z}^{m}$. We denote by $\hat{w}(A, \underline{\theta})$ the supremum of the real numbers $w$ for which, for all sufficiently large positive real numbers $X$, the inequalities (1.1) have an integer solution $\underline{x}$ in $\mathbf{Z}^{m}$.

In the sequel, we consistently use the symbol ^ to indicate that we require a uniform existence of solutions.

The lower bounds

$$
w(A, \underline{\theta}) \geq \hat{w}(A, \underline{\theta}) \geq 0
$$

are then obvious. We define furthermore two homogeneous exponents $w(A)$ and $\hat{w}(A)$ as in (1.1) with $\underline{\theta}={ }^{t}(0, \ldots, 0)$, requiring moreover that the integer solution $\underline{x}$ should be non-zero. The uniform exponent $\hat{w}$ was first introduced and studied by Jarník in the 1930s.

The transposed matrix of a matrix $A$ is denoted by ${ }^{t} A$. Furthermore, $1 /+\infty$ is understood to be 0 . The following result, established in [27, 22], shows that the usual (resp. uniform) inhomogeneous exponents are strongly related to the uniform (resp. usual) homogeneous exponents. 
Theorem 1.3. Let $n$ and $m$ be positive integers and $A$ a real matrix with $n$ rows and $m$ columns. For any $n$-tuple $\underline{\theta}$ of real numbers, we have the lower bounds

$$
w(A, \underline{\theta}) \geq \frac{1}{\hat{w}\left({ }^{t} A\right)} \quad \text { and } \quad \hat{w}(A, \underline{\theta}) \geq \frac{1}{w\left({ }^{t} A\right)},
$$

with equality in (1.2) for almost all $\underline{\theta}$ with respect to the Lebesgue measure on $\mathbf{R}^{n}$.

If the subgroup $G={ }^{t} A \mathbf{Z}^{n}+\mathbf{Z}^{m}$ of $\mathbf{R}^{m}$ generated by the $n$ rows of the matrix $A$ together with $\mathbf{Z}^{m}$ has maximal rank $m+n$, then Kronecker's Theorem asserts that the dual subgroup $\Gamma=A \mathbf{Z}^{m}+\mathbf{Z}^{n}$ of $\mathbf{R}^{n}$ generated by the $m$ columns of $A$ and by $\mathbf{Z}^{n}$ is dense in $\mathbf{R}^{n}$. In this respect, Theorem 1.3 may be viewed as a measure of the density of $\Gamma$. In the case where the rank of $G$ is $<m+n$, we clearly have

$$
\hat{w}\left({ }^{t} A\right)=w\left({ }^{t} A\right)=+\infty \quad \text { and } \quad \hat{w}(A, \underline{\theta})=w(A, \underline{\theta})=0,
$$

for every $n$-tuple $\underline{\theta}$ located outside a discrete family of parallel hyperplanes in $\mathbf{R}^{n}$. The assertion of Theorem 1.3 is then obvious.

Cassels' book [27] remains an invaluable reference for these and related questions.

In the sequel of the text, we restrict our attention to the cases where $A$ is either a row or a column matrix:

$$
A=\left(\xi_{1}, \ldots, \xi_{n}\right) \quad \text { or } \quad A={ }^{t}\left(\xi_{1}, \ldots, \xi_{n}\right) .
$$

This amounts to consider small values of the linear form

$$
\left|x_{0}+x_{1} \xi_{1}+\ldots+x_{n} \xi_{n}\right|, \quad \text { where } x_{0}, x_{1}, \ldots, x_{n} \in \mathbf{Z} \text {, }
$$

or simultaneous approximation to $\xi_{1}, \ldots, \xi_{n}$ by rational numbers with the same denominator, that is, small values of the quantity

$$
\max _{1 \leq j \leq n}\left|x_{0} \xi_{j}-x_{j}\right|, \quad \text { where } x_{0}, x_{1}, \ldots, x_{n} \in \mathbf{Z} \text {. }
$$

Furthermore, among the elements $\underline{\xi}=\left(\xi_{1}, \ldots, \xi_{n}\right)$ in $\mathbf{R}^{n}$, we mainly focus on the points

$$
\left(\xi, \xi^{2}, \ldots, \xi^{n}\right)
$$

whose coordinates are the $n$ first successive powers of a real number $\xi$. However, some of the results stated below hold for a general $n$-tuple $\underline{\xi}$, as will be indicated in due course. This is in particular the case in Sections 6 and 7.

The present paper is organized as follows. In Section 2, we define six exponents of approximation attached to real numbers and give their first properties. We discuss in Section 3 how these exponents are interrelated and study the values taken by these 
exponents in Sections 4 and 5. Intermediate exponents are introduced in Section 6. Parametric geometry of numbers, a deep and powerful, new theory introduced by Schmidt and Summerer [73, 74] and developed by Roy [63], is briefly described in Section 7. Recent results on the existence of real numbers which are badly approximable by algebraic numbers of bounded degree are discussed in Section 8. The final section gathers several open problems and suggestion for further research.

The notation $a \gg_{d} b$ means that $a$ exceeds $b$ times a constant depending only on $d$. When $\gg$ is written without any subscript, it means that the constant is absolute. We write $a \asymp b$ if both $a \gg b$ and $a \ll b$ hold.

\section{Further definitions and first results}

Mahler [49] and Koksma [43] have introduced in the 1930s two classifications of real numbers in terms of their properties of approximation by algebraic numbers. Here and below, the height $H(P)$ of a polynomial $P(X)$ is the maximum of the moduli of its coefficients and the height $H(\alpha)$ of an algebraic number $\alpha$ is the height of its minimal polynomial over $\mathbf{Z}$.

Definition 2.1. Let $n$ be a positive integer. Let $\xi$ be a real number. We denote by $w_{n}(\xi)$ the supremum of the real numbers $w$ for which the inequality

$$
0<|P(\xi)| \leq H(P)^{-w}
$$

is satisfied for infinitely many polynomials $P(X)$ with integer coefficients and degree at most $n$. We denote by $w_{n}^{*}(\xi)$ the supremum of the real numbers $w^{*}$ for which the inequality

$$
0<|\xi-\alpha| \leq H(\alpha)^{-w^{*}-1}
$$

is satisfied for infinitely many algebraic numbers $\alpha$ of degree at most $n$.

The -1 in the exponent of the right hand side of (2.1) can be explained as follows. Let $P(X)$ be an integer polynomial of degree $n \geq 2$ and $\xi$ be a real number not algebraic of degree at most $n$. Observe that $P(\xi) P^{\prime}(\xi) \neq 0$ and

$$
\frac{P^{\prime}(\xi)}{P(\xi)}=\sum_{\alpha: P(\alpha)=0} \frac{1}{\xi-\alpha}
$$

Consequently, if $\alpha$ is the root of $P(X)$ which is closest to $\xi$, then we have

$$
|\xi-\alpha| \leq n\left|P(\xi) / P^{\prime}(\xi)\right|
$$


Since $\left|P^{\prime}(\xi)\right|$ is expected to be roughly equal to $H(P)$ (this is the case unless $P(X)$ has two roots close to $\xi$ ), we expect the estimation

$$
|\xi-\alpha| \ll_{n}|P(\xi)| \cdot H(P)^{-1}
$$

The latter upper bound does not hold when $\left|P^{\prime}(\xi)\right|$ is small, that is, when $P(X)$ has two or more roots close to $\xi$.

The behaviour of the sequences $\left(w_{n}(\xi)\right)_{n \geq 1}$ and $\left(w_{n}^{*}(\xi)\right)_{n \geq 1}$ determines the localisation of $\xi$ in Mahler's and Koksma's classifications, respectively (see Chapter 3 of [15]) ; however, the exact determination of $w_{n}(\xi)$ and $w_{n}^{*}(\xi)$ for a specific real number $\xi$ is usually extremely difficult.

We introduced in [21] four further exponents of approximation. They implicitly appeared previously in articles of Jarník, Davenport and Schmidt, among others.

Definition 2.2. Let $n$ be a positive integer. Let $\xi$ be a real number. We denote by $\lambda_{n}(\xi)$ the supremum of the real numbers $\lambda$ such that the inequality

$$
\max _{1 \leq j \leq n}\left|x_{0} \xi^{j}-x_{j}\right| \leq\left|x_{0}\right|^{-\lambda}
$$

has infinitely many solutions in integers $x_{0}, \ldots, x_{n}$ with $x_{0} \neq 0$.

The three exponents $w_{n}, w_{n}^{*}$ and $\lambda_{n}$ have the common feature to be defined by the existence of infinitely many solutions for some set of Diophantine inequalities. We attach to them three exponents defined by a condition of uniform existence of solutions.

Definition 2.3. Let $n$ be a positive integer and let $\xi$ be a real number. We denote by $\hat{w}_{n}(\xi)$ the supremum of the real numbers $\hat{w}$ such that, for any sufficiently large real number $X$, the inequalities

$$
0<\left|x_{n} \xi^{n}+\ldots+x_{1} \xi+x_{0}\right| \leq X^{-\hat{w}}, \quad \max _{0 \leq j \leq n}\left|x_{j}\right| \leq X
$$

have a solution in integers $x_{0}, \ldots, x_{n}$. We denote by $\hat{w}_{n}^{*}(\xi)$ the supremum of the real numbers $\hat{w}^{*}$ such that, for any sufficiently large real number $X$, there exists an algebraic real number $\alpha$ with degree at most $n$ satisfying

$$
0<|\xi-\alpha| \leq H(\alpha)^{-1} X^{-\hat{w}^{*}} \quad \text { and } \quad H(\alpha) \leq X .
$$

We denote by $\hat{\lambda}_{n}(\xi)$ the supremum of the real numbers $\hat{\lambda}$ such that, for any sufficiently large real number $X$, the inequalities

$$
0<\left|x_{0}\right| \leq X, \quad \max _{1 \leq j \leq n}\left|x_{0} \xi^{j}-x_{j}\right| \leq X^{-\hat{\lambda}}
$$


have a solution in integers $x_{0}, \ldots, x_{n}$.

This survey is mainly devoted to an overview of general results on the six exponents of approximation $w_{n}, w_{n}^{*}, \lambda_{n}, \hat{w}_{n}, \hat{w}_{n}^{*}$ and $\hat{\lambda}_{n}$, whose values are connected through various inequalities. Notice that the exponents 'hat' are uniformly bounded in term of $n$. The exact determination of the upper bounds is an important problem towards the Wirsing Conjecture (see Section 4) or related questions, such as the approximation of transcendental real numbers by algebraic integers. We begin with some easy properties.

Our first result goes back to Khintchine [41].

Proposition 2.4. For any irrational real number $\xi$, we have

$$
\hat{w}_{1}(\xi)=\hat{w}_{1}^{*}(\xi)=\hat{\lambda}_{1}(\xi)=1 .
$$

for every irrational real number $\xi$.

Proof. Let $\xi$ be an irrational real number and $\left(p_{\ell} / q_{\ell}\right)_{\ell \geq 1}$ denote the sequence of its convergents. Let $\ell \geq 4$ and $q$ be integers with $1 \leq q \leq q_{\ell}-1$. Observe that $q_{\ell}-q_{\ell-1} \geq$ $q_{\ell-2} \geq q_{2} \geq 2$. Then, it follows from the theory of continued fractions that

$$
\|q \xi\| \geq\left\|q_{\ell-1} \xi\right\| \geq \frac{1}{q_{\ell}+q_{\ell-1}} \geq \frac{1}{2\left(q_{\ell}-1\right)} .
$$

This shows that, setting $Q=q_{\ell}-1$, the inequality $|q \xi-p| \leq 1 /(2 Q)$ has no solutions in integers $p, q$ with $1 \leq q \leq q_{\ell}-1$. Consequently, $\hat{w}_{1}(\xi)$ is equal to 1 .

We gather in the next theorem several easy results on our six classical exponents of approximation.

Theorem 2.5. For any positive integer $n$ and any real number $\xi$ which is not algebraic of degree $\leq n$, we have

$$
n \leq \hat{w}_{n}(\xi) \leq w_{n}(\xi), \quad \frac{1}{n} \leq \hat{\lambda}_{n}(\xi) \leq \min \left\{1, \lambda_{n}(\xi)\right\}
$$

and

$$
1 \leq \hat{w}_{n}^{*}(\xi) \leq \min \left\{w_{n}^{*}(\xi), \hat{w}_{n}(\xi)\right\} \leq \max \left\{w_{n}^{*}(\xi), \hat{w}_{n}(\xi)\right\} \leq w_{n}(\xi) .
$$

Corollary 5.4 shows that the lower bound $\hat{w}_{n}^{*}(\xi) \geq 1$ in $(2.2)$ is best possible.

Proof. Let $n$ and $\xi$ be as in the statement of the theorem. Let $\alpha$ be a real algebraic number close to $\xi$ and $P_{\alpha}(X)$ the minimal defining polynomial of $\alpha$ over $\mathbf{Z}$. Then, we have $\left|P_{\alpha}(\xi)\right| \ll_{n} H\left(P_{\alpha}\right) \cdot|\xi-\alpha|$ (see e.g. Lemma A.6 of [15]) and we deduce the upper bound $w_{n}^{*}(\xi) \leq w_{n}(\xi)$. The same argumentation gives also that $\hat{w}_{n}^{*}(\xi) \leq \hat{w}_{n}(\xi)$. The upper bounds $\hat{w}_{n}(\xi) \leq w_{n}(\xi), \hat{w}_{n}^{*}(\xi) \leq w_{n}^{*}(\xi)$ and $\hat{\lambda}_{n}(\xi) \leq \lambda_{n}(\xi)$ are consequences of the definitions, while the lower bounds $\hat{w}_{n}(\xi) \geq n$ and $\hat{\lambda}_{n}(\xi) \geq 1 / n$ follow from Dirichlet's box principle (or from Minkowski's theorem). Moreover, we obviously have $\hat{w}_{n}^{*}(\xi) \geq \hat{w}_{1}^{*}(\xi)=1$ and $\hat{\lambda}_{n}(\xi) \leq \hat{\lambda}_{1}(\xi)=1$.

The next theorem was pointed out in [21]. 
Theorem 2.6. For any positive integer $n$ and any real number $\xi$ not algebraic of degree at most $n$, we have

$$
\hat{w}_{n}^{*}(\xi) \geq \frac{w_{n}(\xi)}{w_{n}(\xi)-n+1}
$$

and

$$
w_{n}^{*}(\xi) \geq \frac{\hat{w}_{n}(\xi)}{\hat{w}_{n}(\xi)-n+1}
$$

Wirsing [80] proved a weaker version of (2.3) in which the left-hand side is replaced by the quantity $w_{n}^{*}(\xi)$. His result is also weaker than $(2.4)$, since $\hat{w}_{n}(\xi) \geq w_{n}(\xi)$.

Proof. We follow an argumentation of Wirsing [80]. Let $n \geq 2$ and $\xi$ be as in the statement of the theorem. We first establish (2.3). If $w_{n}(\xi)$ is infinite, then (2.3) reduces to $\hat{w}_{n}^{*}(\xi) \geq 1$, a statement established in Theorem 2.5. Assume that $w_{n}(\xi)$ is finite. Let $\varepsilon>0$ and set $w=w_{n}(\xi)(1+\varepsilon)^{2}$. Minkowski's theorem implies that there exist a constant $c$ and, for any positive real number $H$, a non zero integer polynomial $P(X)$ of degree at most $n$ such that

$$
|P(\xi)| \leq H^{-w}, \quad|P(1)|, \ldots,|P(n-1)| \leq H \quad \text { and } \quad|P(n)| \leq c H^{w-n+1} .
$$

The definition of $w_{n}(\xi)$ and the first inequality of $(2.5)$ show that $H(P)>H^{1+\varepsilon}$ if $H$ is large enough. Consequently, $P(X)$ has some (necessarily real) root in the neighbourhood of each of the points $\xi, 1, \ldots, n-1$. Denoting by $\alpha$ its closest root to $\xi$ and recalling that $H(\alpha) \ll_{n} H(P)$ (see for example Lemma A.3 of [15]), we get

$$
|\xi-\alpha| \ll_{n} \frac{|P(\xi)|}{H(P)} \ll_{n} H(\alpha)^{-1}\left(H^{w-n+1}\right)^{-w /(w-n+1)}
$$

and

$$
H(\alpha) \ll_{n} H(P) \ll_{n} H^{w-n+1} .
$$

Since all of this is true for every sufficiently large $H$, we get $\hat{w}_{n}^{*}(\xi) \geq w /(w-n+1)$. Selecting now $\varepsilon$ arbitrarily close to 0 , we obtain (2.3).

In order to establish (2.4), we may assume that $\hat{w}_{n}(\xi)$ is finite and set $w=$ $\hat{w}_{n}(\xi)(1+\varepsilon)^{2}$. We follow the same argument as in the proof of $(2.3)$. The definition of $\hat{w}_{n}(\xi)$ and the first inequality of (2.5) then show that there exist arbitrarily large values of $H$ for which the polynomial $P(X)$ satisfies $H(P)>H^{1+\varepsilon}$. We conclude that there exist algebraic numbers $\alpha$ of arbitrarily large height with

$$
|\xi-\alpha| \ll_{n} H(\alpha)^{-1-w /(w-n+1)} .
$$

Thus, we get $w_{n}^{*}(\xi) \geq w /(w-n+1)$ and, selecting $\varepsilon$ arbitrarily close to 0 , we obtain (2.4).

The next result shows that if $w_{n}(\xi)=n$ holds, then the values of the five other exponents at the point $\xi$ are known. 
Corollary 2.7. Let $n$ be a positive integer and $\xi$ a real number such that $w_{n}(\xi)=n$. Then we have

$$
w_{n}(\xi)=w_{n}^{*}(\xi)=\hat{w}_{n}(\xi)=\hat{w}_{n}^{*}(\xi)=n
$$

and

$$
\lambda_{n}(\xi)=\hat{\lambda}_{n}(\xi)=\frac{1}{n}
$$

Proof. Equalities (2.6) follow from Theorems 2.5 and 2.6. Khintchine's transference theorem (see Theorem 3.2 below) shows that $w_{n}(\xi)=n$ is equivalent to $\lambda_{n}(\xi)=1 / n$. Combined with Theorem 2.5, this gives (2.7).

Since $w_{n}(\xi)$ is equal to $n$ for almost all real numbers $\xi$, with respect to the Lebesgue measure (this was proved by Sprindžuk [76]), the next result is an immediate consequence of Corollary 2.7.

Theorem 2.8. For almost all (with respect to Lebesgue measure) real numbers $\xi$ and every positive integer $n$, we have

$$
w_{n}(\xi)=w_{n}^{*}(\xi)=\hat{w}_{n}(\xi)=\hat{w}_{n}^{*}(\xi)=n
$$

and

$$
\lambda_{n}(\xi)=\hat{\lambda}_{n}(\xi)=\frac{1}{n}
$$

Before discussing the values taken by our exponents at algebraic points, we recall Liouville's inequality (see Theorem A.1 and Corollary A.2 in [15]).

Theorem 2.9 (Liouville's inequality). Let $\alpha$ and $\beta$ be distinct real algebraic numbers of degree $n$ and $m$, respectively. Then,

$$
|\alpha-\beta| \geq(n+1)^{-m}(m+1)^{-n} H(\alpha)^{-m} H(\beta)^{-n} .
$$

Furthermore, if $P(X)$ is an integer polynomial of degree $n$ which does not vanish at $\beta$, then

$$
|P(\beta)| \geq(n+1)^{-m}(m+1)^{-n} H(P)^{-m+1} H(\beta)^{-n} .
$$

Let $\xi$ be a real algebraic number of degree $d \geq 1$. It follows from Theorem 2.9 that $w_{n}^{*}(\xi) \leq w_{n}(\xi) \leq d-1$ holds for $n \geq 1$. Roth's theorem, which asserts that $w_{1}(\xi)=1$ if $\xi$ is irrational, has been considerably extended by Schmidt [71], who showed that $w_{n}(\xi)=w_{n}^{*}(\xi)=n$ holds for $n \leq d-1$. This enables us to get the values of our six exponents at real algebraic numbers. 
Theorem 2.10. Let $\xi$ be a real algebraic number of degree $d \geq 1$ and let $n$ be a positive integer. We have

$$
w_{n}(\xi)=w_{n}^{*}(\xi)=\hat{w}_{n}(\xi)=\hat{w}_{n}^{*}(\xi)=\min \{n, d-1\}
$$

and

$$
\lambda_{n}(\xi)=\hat{\lambda}_{n}(\xi)=\frac{1}{\min \{n, d-1\}} .
$$

Theorem 2.10 shows that real algebraic numbers of degree greater than $n$ do behave like almost all real numbers, as far as approximation by algebraic numbers of degree less than $n$ is concerned. We may as well consider approximation to complex (non-real) numbers. Quite surprisingly, complex non-real numbers of degree greater than $n$ do not always behave like almost all complex numbers; see [20].

Theorem 2.10 shows that we can focus on the values taken by our exponents at transcendental, real numbers. This motivates the following definition.

Definition 2.11. The spectrum of an exponent of approximation is the set of values taken by this exponent at transcendental real numbers.

We point out an important problem, which will be discussed in the next sections.

Problem 2.12. To determine the spectra of the exponents $w_{n}, w_{n}^{*}, \lambda_{n}, \hat{w}_{n}, \hat{w}_{n}^{*}, \hat{\lambda}_{n}$.

Results towards Problem 2.12 are summarized at the end of Section 5.

\section{Relations between exponents}

We begin this section with an easy result on the difference between the exponents $w_{n}$ and $w_{n}^{*}$.

Theorem 3.1. For any positive integer $n$ and any transcendental real number $\xi$, we have

$$
w_{n}(\xi)-n+1 \leq w_{n}^{*}(\xi) \leq w_{n}(\xi)
$$

and

$$
\hat{w}_{n}(\xi)-n+1 \leq \hat{w}_{n}^{*}(\xi) \leq \hat{w}_{n}(\xi) .
$$

Proof. The right hand side inequalities of (3.1) and (3.2) have been already stated in Theorem 2.5. The left hand side of inequality (3.1) is inequality (3.11) in [15], whose proof also gives the left hand side of inequality (3.2).

It is interesting to note that the left hand side inequality of (3.2) is sharp since there exist real numbers $\xi$ with $\hat{w}_{n}(\xi)=n$ and $\hat{w}_{n}^{*}(\xi)=1$; see Corollary 5.4 below. We do not know if the left hand side inequality of (3.1) is sharp for $n \geq 4$; see Theorem 5.7 below.

We indicate now some transference results linking together the rational simultaneous approximation to $\xi, \ldots, \xi^{n}$ and the smallness of the linear form with coefficients $\xi, \ldots, \xi^{n}$. 
Theorem 3.2. For every integer $n \geq 2$ and every real number $\xi$ which is not algebraic of degree $\leq n$, we have

$$
\frac{1}{n} \leq \frac{w_{n}(\xi)}{(n-1) w_{n}(\xi)+n} \leq \lambda_{n}(\xi) \leq \frac{w_{n}(\xi)-n+1}{n}
$$

and

$$
\frac{1}{n} \leq \frac{\hat{w}_{n}(\xi)-1}{(n-1) \hat{w}_{n}(\xi)} \leq \hat{\lambda}_{n}(\xi) \leq \frac{\hat{w}_{n}(\xi)-n+1}{\hat{w}_{n}(\xi)} .
$$

Proof. The inequalities (3.3) follow direcly from Khintchine's transference principle (cf. Theorem B.5 from [15]), whose proof shows that the same inequalities hold for the uniform exponents; see [37]. The latter result is weaker than inequalities (3.4), which have been recently established by German [35].

Observe that (3.4) with $n=2$ reduces to the following result established by Jarník [37] in 1938; see also [42] for an alternative proof.

Theorem 3.3. For every transcendental real number $\xi$ we have

$$
\hat{\lambda}_{2}(\xi)=1-\frac{1}{\hat{w}_{2}(\xi)} .
$$

Inequalities (3.3) have been recently refined in $[46,24]$ by means of the introduction of uniform exponents.

Theorem 3.4. Suppose $n \geq 2$. The inequalities

$$
\lambda_{n}(\xi) \geq \frac{\left(\hat{w}_{n}(\xi)-1\right) w_{n}(\xi)}{\left((n-2) \hat{w}_{n}(\xi)+1\right) w_{n}(\xi)+(n-1) \hat{w}_{n}(\xi)}
$$

and

$$
\lambda_{n}(\xi) \leq \frac{\left(1-\hat{\lambda}_{n}(\xi)\right) w_{n}(\xi)-n+2-\hat{\lambda}_{n}(\xi)}{n-1}
$$

hold for any transcendental real number $\xi$.

Since $\hat{\lambda}_{n}(\xi) \geq 1 / n$ and $\hat{w}_{n}(\xi) \geq n$, one easily checks that Theorem 3.4 implies (3.3).

The first inequality in the next theorem was established by Davenport and Schmidt [31], while investigating the approximation to a real number by algebraic integers. The second one is a recent result of Schleischitz [67].

Theorem 3.5. For any positive integer $n$ and any transcendental real number $\xi$, we have

$$
w_{n}^{*}(\xi) \geq \frac{1}{\hat{\lambda}_{n}(\xi)} \quad \text { and } \quad \hat{w}_{n}^{*}(\xi) \geq \frac{1}{\lambda_{n}(\xi)} .
$$


It is interesting to note that the combination of (3.4) with the first inequality of Theorem 3.5 gives (2.4).

Theorem 3.5 relates approximation to $\xi$ by algebraic numbers of degree at most $n$ with uniform simultaneous rational approximation to $\xi, \ldots, \xi^{n}$. Further explanations can be found in [26] and in Section 3.6 of [15].

Note that Theorem 3.5 can be compared with the next result, extracted from [22, 27], which is a particular case of Theorem 1.3. If $A$ is the matrix $\left(\xi, \xi^{2}, \ldots, \xi^{n}\right)$ and $\underline{\theta}$ is a real $n$-tuple, we simply write $w_{n}(\xi, \underline{\theta})$ for $w(A, \underline{\theta})$ and $\hat{w}_{n}(\xi, \underline{\theta})$ for $\hat{w}(A, \underline{\theta})$.

Theorem 3.6. For any integer $n \geq 1$, any transcendental real number $\xi$ and any real $n$-tuple $\underline{\theta}$, we have

$$
w_{n}(\xi, \underline{\theta}) \geq \frac{1}{\hat{\lambda}_{n}(\xi)} \quad \text { and } \quad \hat{w}_{n}(\xi, \underline{\theta}) \geq \frac{1}{\lambda_{n}(\xi)} .
$$

We continue with a recent result of Marnat [51] obtained by using the deep result of Roy [63] on the Schmidt-Summerer parametric geometry of numbers ; see Section 7.

Theorem 3.7. For $n \geq 3$, there does not exist any algebraic relation between the exponents $\hat{w}_{n}$ and $\hat{\lambda}_{n}$.

The next results were proved by Schmidt and Summerer $[74,75]$; see also Moshchevitin $[52,53]$.

Theorem 3.8. For any transcendental real number $\xi$ we have

$$
w_{n}(\xi) \geq \hat{w}_{n}(\xi) \frac{(n-1)\left(\hat{w}_{n}(\xi)-1\right)}{1+(n-2) \hat{w}_{n}(\xi)},
$$

for $n \geq 2$, and

$$
w_{3}(\xi) \geq \hat{w}_{3}(\xi) \frac{\sqrt{4 \hat{w}_{3}(\xi)-3}-1}{2} .
$$

The case $n=2$ of Theorem 3.8 was proved by Jarník [39].

Theorem 3.9. For any transcendental real number $\xi$ we have

$$
\lambda_{n}(\xi) \geq \hat{\lambda}_{n}(\xi) \frac{\hat{\lambda}_{n}(\xi)+n-2}{(n-1)\left(1-\hat{\lambda}_{n}(\xi)\right)}
$$

for $n \geq 2$, and

$$
\lambda_{3}(\xi) \geq \hat{\lambda}_{3}(\xi) \frac{\hat{\lambda}_{3}(\xi)+\sqrt{\hat{\lambda}_{3}(\xi)\left(4-3 \hat{\lambda}_{3}(\xi)\right)}}{2\left(1-\hat{\lambda}_{3}(\xi)\right)} .
$$

Actually, Theorems 3.2 to 3.4 and 3.6 to 3.9 are valid for general tuples $\underline{\xi}$, whose coordinates are, together with 1 , linearly independent over $\mathbf{Z}$ (and not only for tuples of the shape $\left(\xi, \xi^{2}, \ldots, \xi^{n}\right)$.) 


\section{Bounds for the exponents}

Let $n$ be a positive integer. As we have seen in Section 2, the Dirichlet Schubfachprinzip (or, if one prefers, Minkowski's theorem) readily implies that $w_{n}(\xi)$ is at least equal to $n$ for any positive integer $n$ and any real number $\xi$ not algebraic of degree $\leq n$. It is a longstanding problem, which was first formulated by Wirsing [80], to decide whether the same result remains true for the quantity $w_{n}^{*}(\xi)$.

Conjecture 4.1 (Wirsing). For any positive integer $n$ and any transcendental real number $\xi$ we have $w_{n}^{*}(\xi) \geq n$.

The seminal paper of Wirsing [80] and the study of his conjecture, which has been up to now confirmed only for $n=1$ (this follows from the theory of continued fractions) and $n=2$ (by Davenport and Schmidt [29]), have motivated many works.

Theorem 4.2. For any real number $\xi$ which is neither rational, nor quadratic, and for any real number $c$ greater than 160/9, there exist infinitely many rational or quadratic real numbers $\alpha$ satisfying

$$
|\xi-\alpha| \leq c \max \left\{1,|\xi|^{2}\right\} H(\alpha)^{-3} .
$$

Theorem 4.2 was proved in [29] (see also [71]). It has been extended by Davenport and Schmidt [30] (up to the value of the numerical constant) as follows.

Theorem 4.3. Let $n \geq 2$ be an integer and let $\xi$ be a real number which is not algebraic of degree at most $n$. Then there exist an effectively computable constant $c$, depending only on $\xi$ and on $n$, an integer $d$ with $1 \leq d \leq n-1$, and infinitely many integer polynomials $P(X)$ of degree $n$ whose roots $\alpha_{1}, \ldots, \alpha_{n}$ can be numbered in such a way that

$$
\left|\left(\xi-\alpha_{1}\right) \ldots\left(\xi-\alpha_{d}\right)\right| \leq c H(P)^{-n-1} .
$$

Theorem 4.2 has recently been improved by Moshchevitin [54] as follows.

Theorem 4.4. For any real number $\xi$ which is neither rational, nor a quadratic irrationality, we have

$$
w_{2}^{*}(\xi) \geq \hat{w}_{2}(\xi)\left(\hat{w}_{2}(\xi)-1\right) \geq 2 .
$$

The proof of Theorem 4.4 combines ideas from [29] with an argument used by Jarník $[38,39]$ in his proof of the case $n=2$ of Theorem 3.8 .

The first statement of Theorem 4.5 was proved by Wirsing [80] and the second one by Bernik and Tishchenko [12]. 
Theorem 4.5. Let $n$ be a positive integer and $\xi$ be a transcendental real number. Then we have

$$
w_{n}^{*}(\xi) \geq \frac{w_{n}(\xi)+1}{2}
$$

and

$$
w_{n}^{*}(\xi) \geq \frac{n}{4}+\frac{\sqrt{n^{2}+16 n-8}}{4} .
$$

For proofs of Theorem 4.5 and related results, the reader may consult Chapter 3 of [15]. Slight improvements on (4.1) have been subsequently obtained by Tishchenko [77, 78], with very technical proofs.

Davenport and Schmidt [31] have given uniform upper bounds for the exponents $\hat{\lambda}_{n}$ and $\hat{w}_{n}$. Some of their results have been subsequently improved by Laurent [44]. For a positive real number $x$, we denote by $\lceil x\rceil$ the smallest integer greater than or equal to $x$. The next theorem expresses results of $[31,44]$ in terms of exponents of approximation.

Theorem 4.6. For any positive integer $n$ and any transcendental real number $\xi$, we have

$$
\hat{\lambda}_{n}(\xi) \leq \frac{1}{\lceil n / 2\rceil} \quad \text { and } \quad \hat{w}_{n}^{*}(\xi) \leq \hat{w}_{n}(\xi) \leq 2 n-1 .
$$

Proof. We only explain how to get the easy estimate $\hat{w}_{n}^{*}(\xi) \leq 2 n-1$. Let $w<\hat{w}_{n}^{*}(\xi)$ be a real number. By definition of $\hat{w}_{n}^{*}$, there exist arbitrarily large integers $H$ such that there are distinct algebraic numbers $\alpha_{1}, \alpha_{2}$ of degree at most $n$ and height at most $H$ such that

$$
\left|\xi-\alpha_{1}\right|<H\left(\alpha_{1}\right)^{-1} H^{-w}, \quad\left|\xi-\alpha_{2}\right|<H\left(\alpha_{2}\right)^{-1} H^{-w}
$$

This implies that $\left|\alpha_{1}-\alpha_{2}\right|<2 H^{-w-1}$, while Theorem 2.9 (Liouville's inequality) gives that $\left|\alpha_{1}-\alpha_{2}\right| \gg_{n} H^{-2 n}$. By combining these two inequalities, we get that $w \leq 2 n-1$. This proves the upper bound $\hat{w}_{n}^{*}(\xi) \leq 2 n-1$. Arguing now with polynomials instead of algebraic numbers, this can be strengthened to $\hat{w}_{n}(\xi) \leq 2 n-1$, as was shown in [31].

Theorem 4.7. For any transcendental real number $\xi$, we have

$$
\hat{\lambda}_{2}(\xi) \leq \frac{\sqrt{5}-1}{2} \quad \text { and } \quad \hat{w}_{2}(\xi) \leq \frac{3+\sqrt{5}}{2}
$$

and both inequalities are best possible. For any transcendental real number $\xi$, we have

$$
\hat{\lambda}_{3}(\xi) \leq(2+\sqrt{5}-\sqrt{7+2 \sqrt{5}}) / 2=0.4245 \ldots
$$

The bound for $\hat{\lambda}_{2}(\xi)$ given in (4.2) was proved in [31] and the one for $\hat{w}_{2}$ in [2], whose authors were at that time not aware of Jarník's result (Theorem 3.3). 
The last assertion of Theorem 4.7 was proved by Roy [61], who improved the estimate $\hat{\lambda}_{3}(\xi) \leq 1 / 2$ given in Theorem 4.6. He further indicated that his upper bound for the exponent is not best possible.

Roy [55, 57] showed that the inequalities (4.2) are sharp. The set of values taken by the exponents $\hat{w}_{2}$ and $\hat{w}_{2}^{*}$ has been studied in [21, 32, 33, 34, 58, 59, 60]. Among other results, we know that the spectrum of $\hat{w}_{2}$ is dense in $[2,(3+\sqrt{5}) / 2]$ and that there exists a real number $c<(3+\sqrt{5}) / 2$ such that the intersection of this spectrum with $[c,(3+\sqrt{5}) / 2]$ is countable.

The next result provides, under a suitable assumption, an upper bound for $\hat{w}_{n}^{*}$ in terms of $w_{m}$, when $m$ is less than $n$. It has not been noticed previously.

Theorem 4.8. Let $m, n$ be positive integers with $1 \leq m \leq n-1$ and $\xi$ be a real number. If $w_{m}(\xi) \geq m+n-1$, then we have

$$
\hat{w}_{n}^{*}(\xi) \leq \frac{m w_{m}(\xi)}{w_{m}(\xi)-n+1}
$$

Proof. It is inspired by that of Proposition 2.1 of [21]. Let $m, n$ and $\xi$ be as in the statement of the theorem. Assume for convenience that $|\xi| \leq 1$. Let $\varepsilon$ be a real number with $0<\varepsilon \leq 1 / 2$. Set $w:=w_{m}(\xi)$. Let $P(X)$ be an integer polynomial of degree at most $m$ and height $H:=H(P)$ large such that

$$
H^{-w-\varepsilon}<|P(\xi)|<H^{-w+\varepsilon}
$$

By using Lemma A.3 from [15], we may assume without any loss of generality that $P(X)$ is irreducible and primitive. Let $v$ be a positive real number and set $X=H^{w /(v(1+\varepsilon))}$. Let $\alpha$ be the root of $P(X)$ which is the closest to $\xi$. If $|\xi-\alpha| \leq H(\alpha)^{-1} H^{-w(1+2 \varepsilon) /(1+\varepsilon)}$, then, by Corollary A.1 of [15], we have

$$
|P(\xi)| \ll_{n}|\xi-\alpha| \cdot H(P) \ll_{n} H^{-w(1+2 \varepsilon / 3)} \ll_{n} H^{-w-4 \varepsilon / 3},
$$

using that $\varepsilon \leq 1 / 2$ and $w \geq 2$. This gives a contradiction to (4.3) if $H$ is large enough. Consequently, we have

$$
|\xi-\alpha|>H(\alpha)^{-1} X^{-v(1+2 \varepsilon)},
$$

provided that $H$ is large enough.

Let $\beta$ be an algebraic number of height at most $X$ and degree at most $n$, which satisfies

$$
|\xi-\beta| \leq H(\beta)^{-1} X^{-v(1+2 \varepsilon)} .
$$

It follows from (4.4) that $\beta \neq \alpha$. Liouville's inequality (Theorem 2.9) then gives

$$
|P(\beta)| \gg_{n} H^{-n+1} H(\beta)^{-m}
$$


By Rolle's theorem and the fact that $|\xi| \leq 1$, we have

$$
|P(\beta)| \leq|\xi-\beta| \cdot \max _{t:|t| \leq 2}\left|P^{\prime}(t)\right|+|P(\xi)| \leq m 2^{m}|\xi-\beta| H+H^{-w+\varepsilon} .
$$

If $H^{-w+\varepsilon} \geq|\xi-\beta| \cdot H$, then (4.6) and (4.7) imply

$$
H(\beta)^{-m} \ll_{n} H^{n-1-w+\varepsilon}
$$

and, since $H(\beta) \leq H^{w /(v(1+\varepsilon))}$, we get

$$
v \leq \frac{m w}{(w+1-n-2 \varepsilon)(1+\varepsilon)},
$$

provided that $H$ is large enough.

If $H^{-w+\varepsilon} \leq|\xi-\beta| \cdot H$, then, by (4.5), (4.6) and (4.7), we get

$$
H(\beta)^{-m+1} H^{-n} \ll_{n} H^{-w(1+2 \varepsilon) /(1+\varepsilon)},
$$

hence,

$$
H^{(m-1) w /(v(1+\varepsilon))} \gg_{n} H^{-n+w(1+2 \varepsilon) /(1+\varepsilon)} .
$$

This implies

$$
v \leq \frac{(m-1) w}{w(1+2 \varepsilon)-n(1+\varepsilon)-\varepsilon},
$$

provided that $H$ is large enough. Consequently, we have $\hat{w}_{n}^{*}(\xi)<v(1+2 \varepsilon)$ as soon as $v$ exceeds the left-hand sides of (4.8) and (4.9). Since $\varepsilon$ can be taken arbitrarily close to 0 , we deduce that

$$
\hat{w}_{n}^{*}(\xi) \leq \max \left\{\frac{m w}{w+1-n}, \frac{(m-1) w}{w-n}\right\} .
$$

Since $w \geq m+n-1$, this maximum is equal to $m w /(w+1-n)$. This ends the proof of the theorem.

We end this section with a recent result of Schleischitz [68].

Theorem 4.9. For any positive integer $n$ and any transcendental real number $\xi$, we have

$$
\hat{\lambda}_{n}(\xi) \leq \max \left\{\frac{1}{n}, \frac{1}{\lambda_{1}(\xi)}\right\} .
$$

Proof. Assume that $n \geq 2$ and that $\xi$ is in $(0,1)$ with $\lambda_{1}(\xi)>1$. Let $q$ be a large positive integer and $v$ be a real number greater than 1 such that $q^{-v}<1 /(4 n q)$ and

$$
\|q \xi\| \leq q^{-v}
$$


Let $p$ be the integer such that $|q \xi-p|=\|q \xi\|$. Without any loss of generality, we may assume that $p$ and $q$ are coprime. Observe that, for $j=1, \ldots, n$, we have

$$
\left|\xi^{j}-\frac{p^{j}}{q^{j}}\right|=\left|\xi-\frac{p}{q}\right| \cdot\left|\xi^{j-1}+\ldots+\left(\frac{p}{q}\right)^{j-1}\right| \leq \frac{2 n}{q^{1+v}} \leq \frac{1}{2 q^{2}}
$$

Let $v^{\prime}$ be a real number with $1<v^{\prime}<\min \{v, n\}$ and set $X=q^{v^{\prime}}$. Let $x$ be a positive integer with $x<X$ and express $x$ in base $q$. There exist integers $b_{0}, b_{1}, \ldots, b_{n-1}$ in $\{0,1, \ldots, q-1\}$ such that

$$
x=b_{0}+b_{1} q+b_{2} q^{2}+\ldots+b_{n-1} q^{n-1} .
$$

Let $u$ be in $\{1,2, \ldots, n\}$ be the smallest index such that $b_{u-1}$ is non-zero. Then,

$$
\left\|x p^{u} / q^{u}\right\|=\left\|b_{u-1} p^{u} / q\right\| \geq 1 / q
$$

Furthermore, it follows from (4.11) and the fact that $v^{\prime}<v$ that

$$
\left|x\left(\xi^{u}-\frac{p^{u}}{q^{u}}\right)\right| \leq \frac{2 n q^{v^{\prime}}}{q^{1+v}}<\frac{1}{2 q}
$$

if $q$ is sufficiently large. Denoting by $y$ the nearest integer to $x \xi^{u}$, the triangle inequality and (4.12) then give

$$
\begin{aligned}
\max _{1 \leq j \leq n}\left\|x \xi^{j}\right\| \geq\left\|x \xi^{u}\right\| & =\left|\left(\frac{x p^{u}}{q^{u}}-y\right)+x\left(\xi^{u}-\frac{p^{u}}{q^{u}}\right)\right| \\
& \geq \frac{1}{q}-\frac{1}{2 q} \geq \frac{1}{2 q}=\frac{1}{2 X^{1 / v^{\prime}}} .
\end{aligned}
$$

If (4.10) is satisfied for arbitrarily large integers $q$, this shows that

$$
\hat{\lambda}_{n}(\xi) \leq \frac{1}{v^{\prime}}
$$

As $v^{\prime}$ can be chosen arbitrarily close to $\min \left\{\lambda_{1}(\xi), n\right\}$, we have proved that

$$
\hat{\lambda}_{n}(\xi) \leq \frac{1}{\min \left\{\lambda_{1}(\xi), n\right\}}=\max \left\{\frac{1}{n}, \frac{1}{\lambda_{1}(\xi)}\right\}
$$

This completes the proof of the theorem.

We point out an immediate corollary of Theorem 4.9 . 
Corollary 4.10. For any positive integer $n$, any transcendental real number $\xi$ with $w_{1}(\xi) \geq n$ satisfies $\hat{w}_{k}(\xi)=k$ for $k=1, \ldots, n$.

Proof. This follows from Theorem 4.9 combined with Theorem 3.2.

\section{Spectra}

This section is mainly devoted to the study of the spectra of the six exponents of approximation defined in Section 2.

We begin with an auxiliary result, extracted from [16], which confirms the existence of real numbers $\xi$ for which $w_{1}(\xi)=w_{n}(\xi)$, for some integer $n \geq 2$.

Theorem 5.1. Let $n \geq 1$ be an integer. For any real number $w \geq 2 n-1$, there exist uncountably many real numbers $\xi$ such that

$$
w_{1}(\xi)=w_{1}^{*}(\xi)=\ldots=w_{n}(\xi)=w_{n}^{*}(\xi)=w .
$$

In particular, the spectra of $w_{n}$ and $w_{n}^{*}$ include the interval $[2 n-1,+\infty]$.

Proof. This is clear for $w=n=1$. Let $w>1$ be a real number. Let $M$ be a large positive integer and consider the real number

$$
\xi_{w}=\left[0 ; 2, M\left\lfloor q_{1}^{w-1}\right\rfloor, M\left\lfloor q_{2}^{w-1}\right\rfloor, M\left\lfloor q_{3}^{w-1}\right\rfloor, \ldots\right],
$$

where $q_{1}=2$ and $q_{j}$ is the denominator of the $j$-th convergent to $\xi_{w}$, that is, of the rational number $p_{j} / q_{j}=\left[0 ; 2, M\left\lfloor q_{1}^{w-1}\right\rfloor, \ldots, M\left\lfloor q_{j-1}^{w-1}\right\rfloor\right]$, for $j \geq 2$. By construction, we have

$$
q_{j+1} \asymp M q_{j}^{w} \quad \text { and } \quad\left|\xi_{w}-\frac{p_{j}}{q_{j}}\right| \asymp \frac{1}{M q_{j}^{w+1}},
$$

for $j \geq 1$. Consequently, we have

$$
w=w_{1}\left(\xi_{w}\right) \leq \ldots \leq w_{n}\left(\xi_{w}\right) .
$$

Using triangle inequalities, it is shown in [16] that, if $M$ is sufficiently large and $w \geq 2 n-1$, then

$$
\left|P\left(\xi_{w}\right)\right| \gg_{n, M} H(P)^{-w}
$$

holds for every polynomial $P(X)$ of degree at most $n$ and sufficiently large height, hence $w_{n}\left(\xi_{w}\right) \leq w$ and the inequalities in (5.1) are indeed equalities. An additional argument is needed to show that $w_{1}^{*}(\xi)=\ldots=w_{n}^{*}(\xi)=w$; see [16] for the complete proof. 
It would be desirable to replace the assumption $w \geq 2 n-1$ in Theorem 5.1 by a weaker one. Actually, the value $2 n-1$ comes from Theorem 2.9 (Liouville's inequality), which is widely used in the proof of Theorem 5.1.

Theorem 5.1 is a key tool to get results on the spectra of various exponents of approximation.

The next result, also established in [16], gives a relationship between the exponents $\lambda_{n}$ and $\lambda_{m}$ when $m$ divides $n$.

Lemma 5.2. For any positive integers $k$ and $n$, and any transcendental real number $\xi$ we have

$$
\lambda_{k n}(\xi) \geq \frac{\lambda_{k}(\xi)-n+1}{n} .
$$

A similar inequality holds between the uniform exponents, but it gives nothing interesting since $\hat{\lambda}_{k}(\xi) \leq 1$ for every integer $k \geq 1$ and every irrational real number $\xi$.

Proof. Let $v$ be a positive real number and $q$ be a positive integer such that

$$
\max _{1 \leq j \leq k}\left|q \xi^{j}-p_{j}\right| \leq q^{-v}
$$

for suitable integers $p_{1}, \ldots, p_{k}$. Let $h$ be an integer with $1 \leq h \leq k n$. Write $h=j_{1}+\ldots+j_{m}$ with $m \leq n$ and $1 \leq j_{1}, \ldots, j_{m} \leq k$. Then,

$$
\left|q^{m} \xi^{h}-p_{j_{1}} \ldots p_{j_{m}}\right| \ll_{m} q^{m-1} q^{-v}
$$

and

$$
\left\|q^{n} \xi^{h}\right\| \ll q^{n-m}\left\|q^{m} \xi^{h}\right\| \ll_{m} q^{n-1-v} \ll_{m}\left(q^{n}\right)^{-(v-n+1) / n},
$$

independently of $h$. This proves the lemma.

We display an immediate consequence of Lemma 5.2.

Corollary 5.3. Let $\xi$ be a real irrational number. Then, $\lambda_{n}(\xi)=+\infty$ holds for every positive integer $n$ if, and only if, $\lambda_{1}(\xi)=+\infty$.

Combined with Theorems 2.5 and 4.8 and Corollary 4.10, Corollary 5.3 allows us to determine the values taken at Liouville numbers (recall that a Liouville number is, by definition, a real number $\xi$ satisfying $w_{1}(\xi)=+\infty$ ) by our six exponents of approximation.

Corollary 5.4. For any positive integer $n$ and any Liouville number $\xi$, we have

$$
w_{n}(\xi)=w_{n}^{*}(\xi)=\lambda_{n}(\xi)=+\infty, \quad \hat{w}_{n}(\xi)=n, \quad \hat{w}_{n}^{*}(\xi)=1, \quad \text { and } \quad \hat{\lambda}_{n}(\xi)=\frac{1}{n} .
$$

The proof of Theorem 5.1 shows how the theory of continued fractions allows us to construct explicitly real numbers $\xi$ having any arbitrarily prescribed value for $\lambda_{1}(\xi)$. The same question for an exponent $\lambda_{n}$ with $n \geq 2$ is not yet solved. At present, the best known result was proved in [16] and is reproduced below. 
Theorem 5.5. Let $n \geq 2$ be an integer and $w \geq n$ be a real number. If the real number $\xi_{w}$ satisfies $w_{1}\left(\xi_{w}\right)=\ldots=w_{n}\left(\xi_{w}\right)=w$, then

$$
\lambda_{n}\left(\xi_{w}\right)=\frac{w-n+1}{n}, \quad \hat{w}_{n}^{*}\left(\xi_{w}\right)=\frac{w}{w-n+1},
$$

and

$$
\hat{w}_{j}\left(\xi_{w}\right)=j, \quad j=1, \ldots, n .
$$

Proof. Let $m \geq 2$ be an integer and $\xi$ be a transcendental real number. Lemma 5.2 with $k=1$ implies the lower bound

$$
\lambda_{m}(\xi) \geq \frac{w_{1}(\xi)-m+1}{m} .
$$

On the other hand, Theorem 3.2 gives the upper bound

$$
\lambda_{m}(\xi) \leq \frac{w_{m}(\xi)-m+1}{m} .
$$

Let $\xi_{w}$ be such that

$$
w_{1}\left(\xi_{w}\right)=\ldots=w_{n}\left(\xi_{w}\right)=w .
$$

Then, the equalities

$$
\lambda_{m}\left(\xi_{w}\right)=\frac{w-m+1}{m}, \quad m=1, \ldots, n,
$$

hold ; in particular,

$$
\lambda_{n}\left(\xi_{w}\right)=\frac{w-n+1}{n},
$$

and this establishes the first statement of the theorem.

Combining Theorem 2.6 with the case $m=1$ of Theorem 4.8 gives

$$
\frac{w_{n}\left(\xi_{w}\right)}{w_{n}\left(\xi_{w}\right)-n+1} \leq \hat{w}_{n}^{*}\left(\xi_{w}\right) \leq \frac{w_{1}\left(\xi_{w}\right)}{w_{1}\left(\xi_{w}\right)-n+1},
$$

thereby proving the second statement of the theorem.

Without any loss of generality, assume that $0<\xi_{w}<1$ and $w>n \geq 2$. Let $\varepsilon$ be a real number satisfying $0<\varepsilon<w-n$. Let $p / q$ be a rational number such that $q \geq 1$, $\operatorname{gcd}(p, q)=1$ and $\left|\xi_{w}-p / q\right|<q^{-1-w+\varepsilon}$. Let $P(X)$ be an integer polynomial of degree $j$ at most $n$ and height at most $q-1$. Observe that $P(p / q)$ is a non-zero rational number satisfying $|P(p / q)| \geq 1 / q^{j}$.

By Rolle's theorem, there exists $t$ lying between $\xi$ and $p / q$ such that

$$
P\left(\xi_{w}\right)=P(p / q)+\left(\xi_{w}-p / q\right) P^{\prime}(t) .
$$

Observe that $\left|P^{\prime}(t)\right| \leq 2^{n} n^{2} q$ and $\left|\xi_{w}-p / q\right| \cdot\left|P^{\prime}(t)\right| \leq 1 /\left(2 q^{n}\right)$, if $q$ is large enough. We then deduce that $\left|P\left(\xi_{w}\right)\right| \geq 1 /\left(2 q^{j}\right)$ if $q$ is large enough. This shows that $\hat{w}_{j}\left(\xi_{w}\right)=j$, as asserted.

Theorem 5.5 allows us to get some information on the spectra of the exponents $\lambda_{n}$ and $\hat{w}_{n}^{*}$. 
Theorem 5.6. For any positive integer $n$, the spectrum of $\lambda_{n}$ includes the interval $[1,+\infty]$, the spectrum of $\hat{w}_{n}^{*}$ includes the interval $[1,2-1 / n]$ and the spectrum of $\hat{w}_{n}-\hat{w}_{n}^{*}$ includes the interval $[n-2+1 / n, n-1]$.

Proof. This has been already proved for $n=1$. If $n \geq 2$, the statement follows from the combination of Theorem 5.1 with Theorem 5.5.

Recall that, by Theorem 3.1, the spectra of $w_{n}-w_{n}^{*}$ and of $\hat{w}_{n}-\hat{w}_{n}^{*}$ are included in $[0, n-1]$, for $n \geq 1$. The first assertion of the next result was proved by Bugeaud and Dujella [19] by means of an explicit construction of families of polynomials with close roots.

Theorem 5.7. For any positive integer $n$, the spectrum of $w_{n}-w_{n}^{*}$ includes the interval

$$
\left[0, \frac{n}{2}+\frac{n-2}{4(n-1)}\right) \text {. }
$$

Moreover, the spectrum of $w_{2}-w_{2}^{*}$ is equal to $[0,1]$ and that of $w_{3}-w_{3}^{*}$ is equal to $[0,2]$.

Explicit examples of real numbers $\xi$ for which $w_{2}(\xi)$ exceeds $w_{2}^{*}(\xi)$ can be found in [18].

Very recently, Schleischitz [68] established that, under some extra assumption, the inequality proved in Lemma 5.2 is indeed an equality.

Theorem 5.8. Let $n$ be a positive integer and $\xi$ be a real number. If $\lambda_{n}(\xi)>1$, then we have

$$
\lambda_{1}(\xi)=n \lambda_{n}(\xi)+n-1
$$

and

$$
\lambda_{j}(\xi)=\frac{n \lambda_{n}(\xi)-j+n}{j}, \quad \hat{\lambda}_{j}(\xi)=\frac{1}{j}, \quad(j=1, \ldots, n) .
$$

Conversely, if $\lambda_{1}(\xi)>2 n-1$, then we have

$$
\lambda_{n}(\xi)=\frac{\lambda_{1}(\xi)-n+1}{n}
$$

and

$$
\hat{\lambda}_{j}(\xi)=\frac{1}{j}, \quad(j=1, \ldots, n) .
$$

Proof. Assume that $\xi$ is in $(0,1)$ and satisfies $\lambda_{n}(\xi)>1$. Let $q$ be a large positive integer and $v$ be a real number greater than 1 such that $q^{-v}<1 /(4 n q)$ and

$$
\max _{1 \leq j \leq n}\left\|q \xi^{j}\right\| \leq q^{-v}
$$


Let $p$ be the integer such that $|q \xi-p|=\|q \xi\|$. Then, $p$ and $q$ may not be coprime, but $p / q$ is a convergent to $\xi$. Let $d$ be the greatest prime factor of $p$ and $q$ and set $p_{0}=p / d$ and $q_{0}=q / d$. Observe that, for $j=1, \ldots, n$, we have

$$
\left|\xi^{j}-\frac{p^{j}}{q^{j}}\right|=\left|\xi-\frac{p}{q}\right| \cdot\left|\xi^{j-1}+\ldots+\left(\frac{p}{q}\right)^{j-1}\right| \leq \frac{2 n}{q^{1+v}} \leq \frac{1}{2 q^{2}} .
$$

Assume that $q<q_{0}^{n}$ and express $q$ in base $q_{0}$. Recalling that $q_{0}$ divides $q$, there exist integers $b_{1}, \ldots, b_{n-1}$ in $\left\{0,1, \ldots, q_{0}-1\right\}$ such that

$$
q=b_{1} q_{0}+b_{2} q_{0}^{2}+\ldots+b_{n-1} q_{0}^{n-1} .
$$

Let $u$ be in $\{2, \ldots, n\}$ be the smallest index such that $b_{u-1}$ is non-zero. Then,

$$
\left\|q p_{0}^{u} / q_{0}^{u}\right\|=\left\|b_{u-1} p_{0}^{u} / q_{0}\right\| \geq 1 / q_{0} .
$$

Furthermore, it follows from (5.6) that

$$
\left|q\left(\xi^{u}-\frac{p^{u}}{q^{u}}\right)\right|=\left|q\left(\xi^{u}-\frac{p_{0}^{u}}{q_{0}^{u}}\right)\right| \leq \frac{1}{2 q} .
$$

Let $y$ be the integer such that $\left|q \xi^{u}-y\right|=\left\|q \xi^{u}\right\|$ and observe that

$$
\left\|q \xi^{u}\right\| \geq\left|y-q \frac{p_{0}^{u}}{q_{0}^{u}}\right|-\left|q\left(\xi^{u}-\frac{p_{0}^{u}}{q_{0}^{u}}\right)\right|,
$$

using the triangle inequality. Combined with (5.7) and (5.8), this gives

$$
\max _{1 \leq j \leq n}\left\|q \xi^{j}\right\| \geq\left\|q \xi^{u}\right\| \geq \frac{1}{q_{0}}-\frac{1}{2 q} \geq \frac{1}{2 q},
$$

a contradiction to (5.5).

Consequently, $b_{1}=\ldots=b_{n-1}=0$ and we have established that $q \geq q_{0}^{n}$ (actually, our proof shows that $q$ must be an integer multiple of $q_{0}^{n}$ ). In particular, we have

$$
d \geq q_{0}^{n-1} .
$$

Since

$$
\left\|q_{0} \xi\right\|=\left|q_{0} \xi-p_{0}\right|=d^{-1}\|q \xi\|,
$$

it follows from (5.5) and (5.9) that

$$
\left\|q_{0} \xi\right\| \leq q_{0}^{-n+1} q_{0}^{-n v}=q_{0}^{-n v-n+1} .
$$


Since $v$ can be taken arbitrarily close to $\lambda_{n}(\xi)$, we deduce that

$$
\lambda_{1}(\xi) \geq n \lambda_{n}(\xi)+n-1 .
$$

Combined with Lemma 5.2, this proves the first statement of the theorem.

In particular, we get $\lambda_{1}(\xi)>2 n-1$, and it follows from Theorem 4.9 that $\hat{\lambda}_{j}(\xi)=1 / j$ for $j=1, \ldots, n$.

Let $j$ be an integer with $2 \leq j \leq n-1$. Since $\lambda_{n}(\xi)>1$, we have $\lambda_{j}(\xi)>1$ and $\lambda_{1}(\xi)=j \lambda_{j}(\xi)+j-1$. Combined with (5.2), this gives $j \lambda_{j}(\xi)=n \lambda_{n}(\xi)-j+n$, as claimed.

If the real number $\xi$ satisfies $\lambda_{1}(\xi)>2 n-1$, then we get by Lemma 5.2 that $\lambda_{n}(\xi)>1$ and (5.3) and (5.4) follow from the first assertions of the theorem.

The condition $\lambda_{n}(\xi)>1$ in the statement of Theorem 5.8 cannot be removed in view of Theorem 4.3 of [16], which confirms the existence of uncountably many real numbers $\xi$ satisfying $\lambda_{n}(\xi)=1$ for every $n \geq 1$. Furthermore, Theorem 4.4 of [16] asserts that, for an arbitrary real number $\lambda$ in $[1,3]$, there exist uncountably many real numbers $\xi$ satisfying $\lambda_{1}(\xi)=\lambda$ and $\lambda_{2}(\xi)=1$.

We display an immediate consequence of Theorem 5.8.

Corollary 5.9. Let $n$ be a positive integer and $\xi$ be a transcendental real number. Then, $\lambda_{n}(\xi)>1$ holds if and only if $\lambda_{1}(\xi)>2 n-1$ holds.

The restriction $w \geq 2 n-1$ in the statement of Theorem 5.1 prevents us to get the whole spectra of the exponents $w_{n}$ and $\lambda_{n}$ by the method described above. Actually, we need the help of metric number theory to determine the whole spectra of the exponents $w_{n}$ and $\lambda_{2}$.

Theorem 5.10. For any positive integer $n$, the spectrum of $w_{n}$ is equal to the whole interval $[n,+\infty]$ and the spectrum of $w_{n}^{*}$ includes the whole interval $[n,+\infty]$.

The first statement of Theorem 5.10 was proved by Bernik [11] and the second one is a result of Baker and Schmidt [5].

We display an immediate consequence of results by Beresnevich, Dickinson, Vaughan and Velani $[10,79]$.

Theorem 5.11. The spectrum of $\lambda_{2}$ is equal to $[1 / 2,+\infty]$.

More is known than the mere statement of Theorems 5.10 and 5.11. Indeed, for an integer $n \geq 1$ and a real number $w \geq n$, the Hausdorff dimension of the set of real numbers $\xi$ for which $w_{n}(\xi)=w$ (resp., $\left.w_{n}^{*}(\xi)=w\right)$ is equal to $(n+1) /(w+1)$. Furthermore, the Hausdorff dimension of the set of real numbers $\xi$ for which $\lambda_{2}(\xi)=\lambda$ is equal to $1 /(1+\lambda)$ if $\lambda \geq 1$ and to $(2-\lambda) /(1+\lambda)$ if $1 / 2 \leq \lambda \leq 1$. 
The spectra of the exponents $\hat{w}_{n}$ and $\hat{\lambda}_{n}$ remain very mysterious for $n \geq 3$, since we can not even exclude that they are, respectively, reduced to $\{n\}$ and $\{1 / n\}$ (recall that, by spectrum, we mean the set of values taken at transcendental points).

For $n=2$, the situation is slightly better. By Jarník's Theorem 3.3, the value of $\hat{w}_{2}$ determines that of $\hat{\lambda}_{2}$, thus it is sufficient to determine the range of $\hat{w}_{2}$; see also below Theorem 4.7.

As for the exponent $\hat{w}_{n}^{*}$, it is likely that its spectrum includes the interval $[1, n]$, but this is not yet proved.

\section{Intermediate exponents}

Let $n \geq 2$ be an integer and $\underline{\xi}$ be a point in $\mathbf{R}^{n}$. In [46], Laurent introduced new exponents $\omega_{n, d}(\underline{\xi})$ (simply denoted by $\omega_{d}(\underline{\xi})$ in [46], since $n$ is fixed throughout that paper) measuring the sharpness of the approximation to $\underline{\xi}$ by linear rational varieties of dimension d. He split the Khintchine transference principle into $n-1$ intermediate estimates which connect the exponents $\omega_{n, d}(\underline{\xi})$ for $d=0,1, \ldots, n-1$ (see also [24]). Actually, Schmidt [70] was the first to investigate the properties of these exponents $\omega_{n, d}$, but he did not introduce them explicitly. We briefly recall their definition and we consider new exponents $w_{n, d}$ defined over $\mathbf{R}$ by restricting $\omega_{n, d}$ to the Veronese curve $\left(x, x^{2}, \ldots, x^{n}\right)$. It is convenient to view $\mathbf{R}^{n}$ as a subset of $\mathbf{P}^{n}(\mathbf{R})$ via the usual embedding $\left(x_{1}, \ldots, x_{n}\right) \mapsto\left(1, x_{1}, \ldots, x_{n}\right)$. We shall identify $\underline{\xi}=\left(\xi_{1}, \ldots, \xi_{n}\right)$ with its image in $\mathbf{P}^{n}(\mathbf{R})$. Denote by $\mathrm{d}$ the projective distance on $\mathbf{P}^{n}(\mathbf{R})$ and, for any real linear subvariety $L$ of $\mathbf{P}^{n}(\mathbf{R})$, set

$$
\mathrm{d}(\underline{\xi}, L)=\min _{P \in L} \mathrm{~d}(\underline{\xi}, P)
$$

the minimal distance between $\underline{\xi}$ and the real points $P$ of $L$. When $L$ is rational over $\mathbf{Q}$, we indicate by $H(L)$ its height, that is the Weil height of any system of Plücker coordinates of $L$. We refer to $[46,24]$ for precise definitions of the projective distance, heights, etc.

Definition 6.1. Let $n \geq 2$ and $d$ be integers with $0 \leq d \leq n-1$. Let $\underline{\xi}$ be in $\mathbf{R}^{n}$. We denote by $\omega_{n, d}(\underline{\xi})$ the supremum of the real numbers $\omega$ for which there exist infinitely many rational linear subvarieties $L \subset \mathbf{P}^{n}(\mathbf{R})$ of dimension $d$ such that

$$
H(L) \mathrm{d}(\underline{\xi}, L) \leq H(L)^{-\omega} .
$$

We denote by $\hat{\omega}_{n, d}(\underline{\xi})$ the supremum of the real numbers $\hat{\omega}$ such that, for every sufficiently large value of $H$, there exists a rational linear subvariety $L \subset \mathbf{P}^{n}(\mathbf{R})$ of dimension $d$ with

$$
H(L) \mathrm{d}(\underline{\xi}, L) \leq H^{-\hat{\omega}} .
$$


If there exists $\xi$ such that $\underline{\xi}=\left(\xi, \xi^{2}, \ldots, \xi^{n}\right)$, then we set $w_{n, d}(\xi)=\omega_{n, d}(\underline{\xi})$ and $\hat{w}_{n, d}(\xi)=\hat{\omega}_{n, d}(\underline{\xi})$.

We observe that the functions $\lambda_{n}$ and $w_{n, 0}$ (resp. $w_{n}$ and $w_{n, n-1}$ ) coincide. The exponents $\hat{\omega}_{n, d}$ were introduced in [23, 64].

The following transference theorem was proved in [70, 46].

Theorem 6.2. Let $n$ be a positive integer. For any non-zero vector $\underline{\xi}$ in $\mathbf{R}^{n+1}$, we have $\omega_{n, 0}(\underline{\xi}) \geq 1 / n$ and

$$
\frac{j \omega_{n, j}(\underline{\xi})}{\omega_{n, j}(\underline{\xi})+j+1} \leq \omega_{n, j-1}(\underline{\xi}) \leq \frac{(n-j) \omega_{n, j}(\underline{\xi})-1}{n-j+1}, \quad j=1, \ldots, n-1,
$$

with the convention that the left-hand side is equal to $j$ if $\omega_{n, j}(\underline{\xi})$ is infinite.

Let the spectrum of the function $\omega_{n, d}$ denote the set of values taken by the exponents $\omega_{n, d}(\underline{\xi})$ when $\underline{\xi}=\left(\xi_{1}, \ldots, \xi_{n}\right)$ ranges over $\mathbf{R}^{n}$, with $1, \xi_{1}, \ldots, \xi_{n}$ linearly independent over the rationals. Using a result of Jarník [36], Laurent [46] established the following statement.

Theorem 6.3. Let $d$ and $n$ be integers with $n \geq 2$ and $0 \leq d \leq n-1$. For every $\omega$ in $[(d+1) /(n-d),+\infty]$, there exists $\underline{\xi}$ such that $\omega_{n, d}(\underline{\xi})=\omega$. Furthermore, $\omega_{n, d}(\underline{\xi})=(d+1) /(n-d)$ for almost all $\underline{\xi}$ in $\mathbf{R}^{n}$.

By means of the numbers $\xi_{w}$ defined in the proof of Theorem 5.1, we get some information on the spectra of the exponents $w_{n, d}$.

Theorem 6.4. For $n \geq 2$ and $0 \leq d \leq n-1$, the spectrum of $w_{n, d}$ contains the whole interval $[(n+d) /(n-d),+\infty]$ and $w_{n, d}(\xi)=(d+1) /(n-d)$ for almost all real numbers $\xi$.

Theorem 6.4 plainly includes the last assertion of Theorem 5.1 and the first assertion of Theorem 5.6.

Proof. We follow the proof of the Corollary from [46], where it is established that, for any $\lambda$ with $1 / n \leq \lambda \leq+\infty$ and for any point $\underline{\xi}$ in $\mathbf{R}^{n}$ such that $\omega_{n, 0}(\underline{\xi})=\lambda$ and $\omega_{n, n-1}(\underline{\xi})=n \lambda+n-1$, we have

$$
\omega_{n, d}(\underline{\xi})=\frac{n \lambda+d}{n-d}, \quad(d=0,1, \ldots, n-1) .
$$

For $w \geq 2 n-1$, the numbers $\xi_{w}$ defined in the proof of Theorem 5.1 satisfy

$$
n \lambda_{n}\left(\xi_{w}\right)=w_{n}\left(\xi_{w}\right)-n+1=w-n+1,
$$

that is,

$$
\omega_{n, n-1}\left(\xi_{w}, \ldots, \xi_{w}^{n}\right)=n \omega_{n, 0}\left(\xi_{w}, \ldots, \xi_{w}^{n}\right)+n-1 .
$$


We then get from (6.1) that

$$
w_{n, d}\left(\xi_{w}\right)=\frac{n \lambda_{n}\left(\xi_{w}\right)+d}{n-d}, \quad(d=0,1, \ldots, n-1) .
$$

The first assertion of the theorem follows since $\lambda_{n}\left(\xi_{w}\right)$ takes every value between 1 and $+\infty$ as $w$ varies from $2 n-1$ to $+\infty$. The second assertion is an immediate consequence of (6.1) and the fact that $n \lambda_{n}(\xi)=w_{n}(\xi)-n+1=1$ holds for almost every real number $\xi$.

We conclude this section by mentioning that Laurent [45] determined the set of values taken by the quadruple of functions $\left(\omega_{2,0}, \omega_{2,1}, \hat{\omega}_{2,0}, \hat{\omega}_{2,1}\right)$ at real points.

\section{Parametric geometry of numbers}

In 2009, Schmidt and Summerer $[73,74]$ introduced a new theory, called the parametric geometry of numbers. They studied the joint behaviour of the $n+1$ successive minima of certain one parameter families of convex bodies in $\mathbf{R}^{n+1}$, as a function of the parameter. They further showed how their results allow them to recover classical inequalities relating various exponents of Diophantine approximation attached to points in $\mathbf{R}^{n}$ and to find new ones. Some aspects of their theory have been simplified and completed by Roy [63], who was then able to derive several spectacular results $[64,65]$.

Let $n$ be a positive integer and $\underline{\xi}$ be a non-zero vector in $\mathbf{R}^{n+1}$. For each real number $Q \geq 1$, we form the convex body

$$
\mathcal{C}_{\underline{\xi}}(Q)=\left\{\underline{x} \in \mathbf{R}^{n+1} ;\|\underline{x}\|_{2} \leq 1,|\underline{x} \cdot \underline{\xi}| \leq Q^{-1}\right\}
$$

where $|\cdot|$ denotes the scalar product and $\|\cdot\|_{2}$ the Euclidean norm. For $j=1, \ldots, n+1$, we denote by $\lambda_{j}\left(\mathcal{C}_{\underline{\xi}}(Q)\right)$ the $j$-th minimum of $\mathcal{C}_{\underline{\xi}}(Q)$, namely the smallest real number $\lambda>0$ such that $\lambda \mathcal{C}_{\underline{\xi}}(\bar{Q})$ contains at least $j$ linearly independent points of $\mathbf{Z}^{n+1}$. Schmidt and Summerer [74] defined

$$
L_{\underline{\xi}, j}(q)=\log \lambda_{j}\left(\mathcal{C}_{\underline{\xi}}\left(\mathrm{e}^{q}\right)\right), \quad q \geq 0,1 \leq j \leq n+1,
$$

and considered the map $\mathbf{L}_{\underline{\xi}}:[0, \infty) \rightarrow \mathbf{R}^{n+1}$ given by

$$
\mathbf{L}_{\underline{\xi}}(q)=\left(L_{\underline{\xi}, 1}(q), \ldots, L_{\underline{\xi}, n+1}(q)\right), \quad q \geq 0 .
$$

They established many properties of this map. For instance, each of its components $L_{\underline{\xi}, j}:[0,+\infty) \rightarrow \mathbf{R}$ is continuous and piecewise linear with slopes 0 and 1 . Schmidt and Summerer showed that each function $\mathbf{L}_{\underline{\xi}}$ can be approximated up to bounded difference by functions from a certain class, and Roy [63] showed that the same property holds within a simpler class. 
For $j=1, \ldots, n+1$, Roy [64] also introduced

$$
\underline{\psi}_{j}(\underline{\xi})=\liminf _{q \rightarrow+\infty} \frac{L_{\underline{\xi}, 1}(q)+\cdots+L_{\underline{\xi}, j}(q)}{q} \quad \text { and } \quad \bar{\psi}_{j}(\underline{\xi})=\limsup _{q \rightarrow+\infty} \frac{L_{\underline{\xi}, 1}(q)+\cdots+L_{\underline{\xi}, j}(q)}{q} .
$$

The following result, established in [64], connects these quantities to those from the previous section.

Proposition 7.1. Let $n$ be a positive integer and $\underline{\xi}=\left(1, \xi_{1}, \ldots, \xi_{n}\right)$ be a vector in $\mathbf{R}^{n+1}$. For $j=0, \ldots, n-1$, we have

$$
\omega_{n, j}\left(\left(\xi_{1}, \ldots, \xi_{n}\right)\right)=\frac{1}{\underline{\psi}_{n-j}(\underline{\xi})}-1 \quad \text { and } \quad \hat{\omega}_{n, j}\left(\left(\xi_{1}, \ldots, \xi_{n}\right)\right)=\frac{1}{\bar{\psi}_{n-j}(\underline{\xi})}-1 .
$$

We quote below the main result of [64] and observe that it implies the first statement of Theorem 6.3.

Theorem 7.2. Let $n$ be a positive integer. For any $\omega_{0}, \ldots, \omega_{n-1} \in[0,+\infty]$ satisfying $\omega_{0} \geq 1 / n$ and

$$
\frac{j \omega_{n, j}}{\omega_{n, j}+j+1} \leq \omega_{n, j-1} \leq \frac{(n-j) \omega_{n, j}-1}{n-j+1}, \quad 1 \leq j \leq n-1,
$$

there exists a point $\underline{\xi} \in \mathbf{R}^{n}$, whose coordinates are, together with 1, linearly independent over $\mathbf{Q}$, such that

$$
\omega_{n, j}(\underline{\xi})=\omega_{j} \quad \text { and } \quad \hat{\omega}_{n, j}(\underline{\xi})=\frac{j+1}{n-j}, \quad 0 \leq j \leq n-1 .
$$

Furthermore, the point of view of parametric geometry of numbers has led Schmidt and Summerer to introduce the following exponents of approximations.

Definition 7.3. Let $\underline{\xi}=\left(\xi_{1}, \ldots, \xi_{n}\right)$ be in $\mathbf{R}^{n}$. For $i=1, \ldots, n+1$, we denote by $\lambda_{i, n}(\underline{\xi})$ (resp. $\hat{\lambda}_{i, n}(\underline{\xi})$ ) the supremum of the real numbers $\lambda$ such that the system of inequalities

$$
0<\left|x_{0}\right| \leq X, \quad \max _{1 \leq j \leq n}\left|x_{0} \xi_{j}-x_{j}\right| \leq X^{-\lambda}
$$

has $i$ linearly independent solutions $\left(x_{0}, x_{1}, \ldots, x_{n}\right)$ in $\mathbf{Z}^{n}$ for arbitrarily large $X$ (resp. for every sufficiently large $X$ ).

Schmidt and Summerer [74] observed that $\lambda_{1, n}(\underline{\xi})=\lambda_{n}(\underline{\xi}), \hat{\lambda}_{1, n}(\underline{\xi})=\hat{\lambda}_{n}(\underline{\xi})$, $\lambda_{n+1, n}(\underline{\xi})=1 / \hat{w}_{n}(\underline{\xi})$ and $\hat{\lambda}_{n+1, n}(\underline{\xi})=1 / w_{n}(\underline{\xi})$, by Mahler's theorem on polar reciprocal bodies [50].

These exponents have been studied by Schleischitz [66, 69]. 


\section{Real numbers which are badly approximable by algebraic numbers}

This short section is devoted to Problems 24 and 25 of [15], which were solved by Badziahin and Velani [4], for $n=2$, and by Beresnevich [8], for $n \geq 3$.

Recall that an irrational real number $\xi$ is called a badly approximable number if there exists a positive real number $c$ such that

$$
|q x-p|>\frac{c}{|q|}, \quad \text { for every } p, q \text { in } \mathbf{Z} \text { with } q \neq 0 .
$$

This notion can be extended as follows.

Definition 8.1. Let $n$ be a positive integer. A real number $\xi$ is called n-badly approximable if there exists a positive constant $c(\xi, n)$ such that

$$
|P(\xi)| \geq c(\xi, n) H(P)^{-n} \quad \text { for any integer polynomial } P(X) \text { of degree } \leq n .
$$

Observe that it follows from Liouville's Inequality (Theorem 2.9) that, for any positive integer $n$, any real algebraic number of degree $n+1$ is $n$-badly approximable.

Davenport [28] asked whether there exist 2-badly approximable transcendental real numbers. His question remained open for nearly fifty years, until it was finally solved by Badziahin and Velani [4], using an intricate construction inspired by their proof [3] of a conjecture of Schmidt. Their result was subsequently extended a few years later by Beresnevich [8], who confirmed the existence of $n$-badly approximable transcendental real numbers, for every given positive integer $n$. For $n=2$, his proof differs greatly from that of [4].

A closely related problem deals with transcendental numbers badly approximable by algebraic numbers of degree at most equal to some integer $n$. As well, it has been solved by Badziahin and Velani [4], for $n=2$, and by Beresnevich [8], for $n \geq 3$.

Theorem 8.2. Let $n$ be a positive integer. There exist transcendental real numbers $\xi$ which are $n$-badly approximable and for which there exist positive real numbers $c_{1}(\xi, n)$ and $c_{2}(\xi, n)$ such that

$$
|\xi-\alpha| \geq c_{1}(\xi, n) H(\alpha)^{-n-1}, \quad \text { for any real algebraic number } \alpha \text { of degree } \leq n,
$$

and

$|\xi-\alpha| \leq c_{2}(\xi, n) H(\alpha)^{-n-1}, \quad$ for infinitely many real algebraic numbers $\alpha$ of degree $\leq n$.

Moreover, the set of real numbers with this property has full Hausdorff dimension.

Actually, Beresnevich [8] established that any intersection of finitely many of the sets arising in Theorem 8.2 has full Hausdorff dimension. 


\section{Open problems}

In Chapter 10 of [15] we listed several open questions. As we have already seen above, some of them have now been solved. We gather below some of the still open problems mentioned in [15], and add a few supplementary ones.

We begin with the conjecture of Wirsing [80] dealing with the approximation of real transcendental numbers by real algebraic numbers of bounded degree. This celebrated open problem has motivated many works in this area.

Problem 9.1 (Wirsing's Conjecture). For any integer $n \geq 1$ and for any real transcendental number $\xi$, we have $w_{n}^{*}(\xi) \geq n$.

We may even ask for a stronger version of Wirsing's conjecture, namely whether, for any positive integer $n$ and any real transcendental number $\xi$, there exist a constant $c(\xi, n)$ and infinitely many real algebraic numbers $\alpha$ of degree less than or equal to $n$ such that

$$
|\xi-\alpha| \leq c(\xi, n) H(\alpha)^{-n-1}
$$

Davenport and Schmidt [29] gave a positive answer to this question in the case $n=2$ (and Theorem 8.2 implies that their result is best possible up to the value of the numerical constant), but we do not know if we can fix the exact degree of the approximants instead of just an upper bound for it.

Problem 9.2. For any integer $n \geq 2$ and any real transcendental number $\xi$, there exist a constant $c(\xi, n)$ and infinitely many real algebraic numbers $\alpha$ of degree exactly $n$ such that

$$
|\xi-\alpha| \leq c(\xi, n) H(\alpha)^{-n-1}
$$

Results of Roy $[56,57]$ could speak in favour of the existence of transcendental numbers which do not satisfy the conclusion of Problem 9.2, even for $n=2$.

The next problem was called the 'main problem' in [15].

Problem 9.3. Let $\left(w_{n}\right)_{n \geq 1}$ and $\left(w_{n}^{*}\right)_{n \geq 1}$ be two non-decreasing sequences in $[1,+\infty]$ such that

$$
n \leq w_{n}^{*} \leq w_{n} \leq w_{n}^{*}+n-1, \quad \text { for any } n \geq 1
$$

Then there exists a real transcendental number $\xi$ such that

$$
w_{n}(\xi)=w_{n} \quad \text { and } \quad w_{n}^{*}(\xi)=w_{n}^{*} \quad \text { for any } n \geq 1 .
$$

Since Problem 9.3 does not take the exponents $\lambda_{n}$ into account, we propose a more general formulation. 
Problem 9.4. For $n \geq 2$, determine the joint spectrum of the triple of exponents $\left(w_{n}, w_{n}^{*}, \lambda_{n}\right)$, that is, the set of triples $\left(w_{n}(\xi), w_{n}^{*}(\xi), \lambda_{n}(\xi)\right)$, when $\xi$ goes through the set of transcendental real numbers.

Theorem 3.2 shows how the exponents $\lambda_{n}$ are related to the exponents $w_{n}$ by means of a transference theorem.

Problem 9.5. Let $n \geq 2$ be an integer, $\lambda_{n} \geq 1 / n$ and $w_{n} \geq n$ be real numbers satisfying

$$
\frac{w_{n}}{(n-1) w_{n}+n} \leq \lambda_{n} \leq \frac{w_{n}-n+1}{n} .
$$

There exist real numbers $\xi$ such that $w_{n}(\xi)=w_{n}$ and $\lambda_{n}(\xi)=\lambda_{n}$.

Corollary 2.7 asserts that $w_{n}^{*}(\xi)=n$ holds if $w_{n}(\xi)=n$, but the converse is an open question.

Problem 9.6. For any positive integer $n$, we have $w_{n}(\xi)=n$ if $w_{n}^{*}(\xi)=n$.

We now turn to uniform exponents.

Problem 9.7. For any integer $n \geq 3$ and any real transcendental number $\xi$, we have $\hat{w}_{n}(\xi)=n$. At least, obtain a better upper bound than $\hat{w}_{n}(\xi) \leq 2 n-1$.

Approximation by algebraic integers (that is, by algebraic numbers, whose minimal defining polynomial over $\mathbf{Z}$ is monic) has been first studied by Davenport and Schmidt [31]. Roy [56] proved that there exist real numbers $\xi$ which are very badly approximable by quadratic integers, in the sense that there exists $c>0$ such that

$$
|\xi-\alpha|>c H(\alpha)^{-(1+\sqrt{5}) / 2}
$$

for every real quadratic number $\alpha$.

The next problem is the analogue of Wirsing's conjecture for approximation by algebraic integers.

Problem 9.8. For any integer $n \geq 4$, any positive real number $\varepsilon$, and any real transcendental number $\xi$, there exist a constant $c(\xi, n, \varepsilon)$ and infinitely many real algebraic integers $\alpha$ of degree less than or equal to $n$ such that

$$
|\xi-\alpha| \leq c(\xi, n, \varepsilon) H(\alpha)^{-n+\varepsilon}
$$

In view of auxiliary results from [31], the answer to Problem 9.8 is positive for some integer $n \geq 4$ if one can prove that any real transcendental number $\xi$ satisfies $\hat{w}_{n-1}(\xi)=n-1$.

Despite the recent progress made in $[4,8]$, the following problem remains open. 
Problem 9.9. There exist a real transcendental number $\xi$ and a sequence $(c(\xi, n))_{n \geq 1}$ of positive real numbers such that

$$
|P(\xi)| \geq c(\xi, n) H(P)^{-n} \quad \text { for any integer } n \text { and any polynomial } P(X) \text { of degree } \leq n \text {. }
$$

It is likely that the answer to Problem 9.9 is positive and that, moreover, the set of real numbers $\xi$ with this property has full Hausdorff dimension.

We continue with a problem proposed by Schleischitz [68], which corrects and refines a problem posed in [16].

Problem 9.10. Let $m, n$ be integers with $1 \leq n \leq m$. Does the inequality

$$
\lambda_{m}(\xi) \geq \frac{n \lambda_{n}(\xi)-m+n}{m}
$$

hold for every transcendental real number $\xi$ ?

The next problem extends a question posed by Beresnevich, Dickinson, and Velani [9] in the case of (simultaneous) rational approximation.

Problem 9.11. Let $n$ be a positive integer and let $\tau>1$ be real. Is the set of real numbers $\xi$ for which there exists a positive constant $c(\xi)$ such that

$$
|\xi-\alpha| \leq H(\alpha)^{-\tau(n+1)}, \quad \text { for infinitely many algebraic numbers } \alpha \text { of degree } \leq n,
$$

and

$$
|\xi-\alpha| \geq c(\xi) H(\alpha)^{-\tau(n+1)}, \quad \text { for every algebraic number } \alpha \text { of degree } \leq n,
$$

non-empty? If yes, determine its Hausdorff dimension.

Problem 9.11 has been solved [14] when $n=1$. One may also replace the approximation functions $x \mapsto x^{-\tau(n+1)}$ by more general non-increasing functions $\Psi$.

Problems 9.12 and 9.13 deal with metrical results.

Problem 9.12. Let $n \geq 2$ be an integer. Let $\lambda_{n}$ be a real number with $\lambda_{n} \geq 1 / n$. Determine the Hausdorff dimension of the set of real numbers $\xi$ such that $\lambda_{n}(\xi)=\lambda_{n}$.

Problem 9.13. Determine the Hausdorff dimension of the set of real numbers $\xi$ such that $\hat{w}_{2}(\xi)>2\left(\operatorname{resp} . \hat{w}_{2}^{*}(\xi)>2\right)$.

We continue with two specific questions on exponents of uniform approximation. 
Problem 9.14. Does there exist $\xi$ such that $\hat{w}_{2}(\xi)>2$ and $\hat{w}_{2}(\xi)>\hat{w}_{2}^{*}(\xi)$ ?

Problem 9.15. The spectrum of $\hat{w}_{2}^{*}$ includes the interval $[1,2]$.

Let $m \geq 2$ be an integer. According to LeVeque [47], a real number $\xi$ is a $U_{m}$-number if $w_{m}(\xi)$ is infinite and $w_{m-1}(\xi)$ is finite. Furthermore, the $U_{1}$-numbers are precisely the Liouville numbers.

It is proved in [1] (see also Section 7.6 of [15]) that, for any integer $m \geq 2$, there exist uncountably many real $U_{m}$-numbers $\xi$ with

$$
w_{n}^{*}(\xi) \leq m+n-1, \quad \text { for } n=1, \ldots, m-1 .
$$

Schmidt [72] showed that $w_{n}^{*}(\xi)$ can be replaced by $w_{n}(\xi)$ in $(9.1)$.

Problem 9.16. Let $m \geq 2$ be an integer. There exist real $U_{m}$-numbers $\xi$ satisfying $w_{n}(\xi)=n$, for $n=1, \ldots, m-1$.

Corollary 5.4 shows that the values taken by our exponents of approximation at $U_{1^{-}}$ numbers are known.

Problem 9.17. Let $m$ and $n$ be integers with $n \geq m \geq 2$. Study the values taken by the exponents of approximation $\lambda_{n}, \hat{w}_{n}^{*}, \ldots$ at $U_{m}$-numbers.

Among many questions concerning the exponents $w_{n, d}$ and $\hat{w}_{n, d}$ defined in Section 6 , let us point out the following three ones.

Problem 9.18. Let $d$ and $n$ be integers with $n \geq 2$ and $0 \leq d \leq n-1$. Find a real number $C_{n, d}$ as small as possible such that every transcendental real number $\xi$ satisfies $\hat{w}_{n, d}(\xi) \leq C_{n, d}$.

Problem 9.19. Let $d$ and $n$ be integers with $n \geq 2$ and $0 \leq d \leq n-1$. Is the spectrum of the function $w_{n, d}$ equal to $[(d+1) /(n-d),+\infty]$ ?

A positive answer of Problem 9.19 would (probably) follow from the resolution of the next problem.

Problem 9.20. Let $d$ and $n$ be integers with $n \geq 2$ and $0 \leq d \leq n-1$. Let $w$ be a real number satisfying $w>(d+1) /(n-d)$. Determine the Hausdorff dimension of the sets

$$
\left\{\underline{\xi} \in \mathbf{R}^{n}: \omega_{n, d}(\underline{\xi}) \geq w\right\}
$$

and

$$
\left\{\xi \in \mathbf{R}: w_{n, d}(\xi) \geq w\right\}
$$

Throughout this survey, we have focus our attention on approximation to real numbers. However, we may as well consider approximation to complex numbers or to 
p-adic numbers; see the references given in Chapter 9 of [15] and the works $[20,81,17$, $13,7,40,25]$.

There are as well several recent papers on uniform Diophantine approximation on curves in $\mathbf{R}^{2}$; see $[48,62,6]$.

\section{References}

[1] K. Alniaçik, Y. Avci, and Y. Bugeaud, On $U_{m}$ numbers with small transendence measure, Acta Math. Hungar. 99 (2003), 271-277.

[2] B. Arbour and D. Roy, A Gel'fond type criterion in degree two, Acta Arith. 111 (2004), 97-103.

[3] D. Badziahin, A. Pollington, and S. Velani, On a problem in simultaneous diophantine approximation: Schmidt's conjecture, Ann. of Math. 174 (2011), 1837-1883.

[4] D. Badziahin and S. Velani, Badly approximable points on planar curves and a problem of Davenport, Math. Ann. 359 (2014), 969-1023.

[5] A. Baker and W. M. Schmidt, Diophantine approximation and Hausdorff dimension, Proc. London Math. Soc. 21 (1970), 1-11.

[6] G. Batzaya, On simultaneous approximation to powers of a real number by rational numbers, J. Number Theory 147 (2015), 141-155.

[7] P. Bel, Approximation simultanée d'un nombre v-adique et de son carré par des nombres algébriques, J. Number Theory 133 (2013), 3362-3380.

[8] V. Beresnevich, Badly approximable points on manifolds. Preprint.

[9] V. V. Beresnevich, H. Dickinson, and S. L. Velani, Sets of exact 'logarithmic order' in the theory of Diophantine approximation, Math. Ann. 321 (2001), 253-273.

[10] V. Beresnevich, D. Dickinson and S. L. Velani, Diophantine approximation on planar curves and the distribution of rational points, with an appendix by R.C. Vaughan: 'Sums of two squares near perfect squares', Ann. of Math. 166 (2007), 367-426.

[11] V. I. Bernik, Application of the Hausdorff dimension in the theory of Diophantine approximations, Acta Arith. 42 (1983), 219-253 (in Russian). English transl. in Amer. Math. Soc. Transl. 140 (1988), 15-44.

[12] V. I. Bernik and K. Tishchenko, Integral polynomials with an overfall of the coefficient values and Wirsing's problem, Dokl. Akad. Nauk Belarusi 37 (1993), no. 5, 9-11 (in Russian). 
[13] N. Budarina, Y. Bugeaud, D. Dickinson, and H. O'Donnell, On simultaneous rational approximation to a p-adic number and its integral powers, Proc. Edinb. Math. Soc. 54 (2011), 599-612.

[14] Y. Bugeaud, Sets of exact approximation order by rational numbers, Math. Ann. 327 (2003), 171-190.

[15] Y. Bugeaud, Approximation by algebraic numbers, Cambridge Tracts in Mathematics, Cambridge, 2004.

[16] Y. Bugeaud, On simultaneous rational approximation to a real number and its integral powers, Ann. Inst. Fourier (Grenoble) 60 (2010), 2165-2182.

[17] Y. Bugeaud, On simultaneous uniform approximation to a p-adic number and its square, Proc. Amer. Math. Soc. 138 (2010), 3821-3826.

[18] Y. Bugeaud, Continued fractions with low complexity: Transcendence measures and quadratic approximation, Compos. Math. 148 (2012), 718-750.

[19] Y. Bugeaud and A. Dujella, Root separation for irreducible integer polynomials, Bull. Lond. Math. Soc. 43 (2011), 1239-1244.

[20] Y. Bugeaud and J.-H. Evertse, Approximation of complex algebraic numbers by algebraic numbers of bounded degree, Ann. Scuola Normale Superiore di Pisa 8 (2009), 333-368.

[21] Y. Bugeaud and M. Laurent, Exponents of Diophantine Approximation and Sturmian Continued Fractions, Ann. Inst. Fourier (Grenoble) 55 (2005), 773-804.

[22] Y. Bugeaud and M. Laurent, Exponents of homogeneous and inhomogeneous Diophantine Approximation, Moscow Math. J. 5 (2005), 747-766.

[23] Y. Bugeaud and M. Laurent, Exponents of Diophantine approximation. In: Diophantine Geometry Proceedings, Scuola Normale Superiore Pisa, Ser. CRM, vol. 4, 2007, $101-121$.

[24] Y. Bugeaud and M. Laurent, On transfer inequalities in Diophantine approximation, II, Math. Z. 265 (2010), 249-262.

[25] Y. Bugeaud and T. Pejkovic, Quadratic approximation in $\mathbf{Q}_{p}$, Intern. J. Number Theory 11 (2015), 193-209.

[26] Y. Bugeaud et O. Teulié, Approximation d'un nombre réel par des nombres algébriques de degré donné, Acta Arith. 93 (2000), 77-86.

[27] J. W. S. Cassels, An introduction to Diophantine Approximation, Cambridge Tracts in Math. and Math. Phys., vol. 99, Cambridge University Press, 1957. 
[28] H. Davenport, A note on Diophantine approximation. II, Mathematika 11 (1964), $50-58$.

[29] H. Davenport and W. M. Schmidt, Approximation to real numbers by quadratic irrationals, Acta Arith. 13 (1967), 169-176.

[30] H. Davenport and W. M. Schmidt, A theorem on linear forms, Acta Arith. 14 (1967/1968), 209-223.

[31] H. Davenport and W. M. Schmidt, Approximation to real numbers by algebraic integers, Acta Arith. 15 (1969), 393-416.

[32] S. Fischler, Spectres pour l'approximation d'un nombre réel et de son carré, C. R. Acad. Sci. Paris 339 (2004), 679-682.

[33] S. Fischler, Palindromic prefixes and episturmian words, J. Combin. Theory Ser. A 113 (2006), 1281-1304.

[34] S. Fischler, Palindromic prefixes and Diophantine approximation, Monatsh. Math. 151 (2007), 11-37.

[35] O. N. German, On Diophantine exponents and Khintchine's transference principle, Mosc. J. Comb. Number Theory 2 (2012), 22-51.

[36] V. Jarník, Über einen Satz von A. Khintchine, 2. Mitteilung, Acta Arith. 2 (1936), $1-22$.

[37] V. Jarník, Zum Khintchineschen "Übertragungssatz", Trav. Inst. Math. Tbilissi 3 (1938), 193-212.

[38] V. Jarník, Une remarque sur les approximations diophantiennes linéaires, Acta Sci. Math. Szeged 12 (1950), 82-86.

[39] V. Jarník, Contribution à la théorie des approximations diophantiennes linéaires et homogènes, Czechoslovak Math. J. 4 (1954), 330-353 (in Russian, French summary).

[40] G. Kekec, On Mahler's p-adic $U_{m}$-numbers, Bull. Aust. Math. Soc. 88 (2013), 44-50.

[41] A. Ya. Khintchine, Über eine Klasse linearer diophantischer Approximationen, Rendiconti Circ. Mat. Palermo 50 (1926), 170-195.

[42] A. Ya. Khintchine, On some applications of the method of the additional variable, Uspehi Matem. Nauk 3, (1948), 188-200 (in Russian). English translation: Amer. Math. Soc. Translation (1950), no. 18, 14 pp.

[43] J. F. Koksma, Über die Mahlersche Klasseneinteilung der transzendenten Zahlen und die Approximation komplexer Zahlen durch algebraische Zahlen, Monatsh. Math. Phys. 48 (1939), 176-189. 
[44] M. Laurent, Simultaneous rational approximation to the successive powers of a real number, Indag. Math. 11 (2003), 45-53.

[45] M. Laurent, Exponents of Diophantine approximation in dimension two, Canad. J. Math. 61 (2009), 165-189.

[46] M. Laurent, On transfer inequalities in Diophantine approximation. In: Analytic number theory, 306-314, Cambridge Univ. Press, Cambridge, 2009.

[47] W. J. LeVeque, On Mahler's U-numbers, J. London Math. Soc. 28 (1953), 220-229.

[48] S. Lozier and D. Roy, Simultaneous approximation to a real number and to its cube by rational numbers, Acta Arith. 156 (2012), 39-73.

[49] K. Mahler, Zur Approximation der Exponentialfunktionen und des Logarithmus. I, II, J. reine angew. Math. 166 (1932), 118-150.

[50] K. Mahler, Ein Übertreibungsprinzip für konvexe Körper, Časopis Pěst. Mat. Fyz. 68 (1939), 93-102.

[51] A. Marnat, About Jarnik-type relations in higher dimension. In preparation.

[52] N. G. Moshchevitin, Singular Diophantine systems of A. Ya. Khinchin and their application, Uspekhi Mat. Nauk 65 (2010), 43-126 ; English translation in Russian Math. Surveys 65 (2010), 433-511.

[53] N. Moshchevitin, Exponents for three-dimensional simultaneous Diophantine approximations, Czechoslovak Math. J. 62 (2012), 127-137.

[54] N. Moshchevitin, A note on two linear forms, Acta Arith. 162 (2014), 43-50.

[55] D. Roy, Approximation simultanée d'un nombre et son carré, C. R. Acad. Sci. Paris 336 (2003), 1-6.

[56] D. Roy, Approximation to real numbers by cubic algebraic numbers, II, Ann. of Math. 158 (2003), 1081-1087.

[57] D. Roy, Approximation to real numbers by cubic algebraic numbers, I, Proc. London Math. Soc. 88 (2004), 42-62.

[58] D. Roy, Diophantine approximation in small degree, Number Theory, 269-285, CRM Proc. Lecture Notes 36, Amer. Math. Soc., Providence, RI, 2004.

[59] D. Roy, On two exponents of approximation related to a real number and its square, Canad. J. Math. 59 (2007), 211-224.

[60] D. Roy, On the continued fraction expansion of a class of numbers. In: Diophantine approximation, Festschrift for Wolfgang Schmidt, Developments in Math. vol. 16, Eds: H. P. Schlickewei, K. Schmidt and R. Tichy, Springer-Verlag, 2008, 347-361. 
[61] D. Roy, On simultaneous rational approximations to a real number, its square, and its cube, Acta Arith. 133 (2008), 185-197.

[62] D. Roy, Rational approximation to real points on conics, Ann. Inst. Fourier (Grenoble) 63 (2013), 2331-2348.

[63] D. Roy, On Schmidt and Summerer parametric geometry of numbers, Ann. of Math. To appear.

[64] D. Roy, Spectrum of the exponents of best rational approximation. Preprint.

[65] D. Roy, Construction of points realizing the regular systems of Wolfgang Schmidt and Leonard Summerer, J. Théor. Nombres Bordeaux. To appear.

[66] J. Schleischitz, Diophantine approximation and special Liouville numbers, Commun. Math. 21 (2013), 39-76.

[67] J. Schleischitz, Two estimates concerning classical Diophantine approximation constants, Publ. Math. Debrecen 84 (2014), 415-437.

[68] J. Schleischitz, On the spectrum of Diophantine approximation constants. Preprint. arXiv:1409.1472.

[69] J. Schleischitz, On approximation constants for Liouville numbers. Preprint. arXiv:1409.1396.

[70] W. M. Schmidt, On heights of algebraic subspaces and diophantine approximations, Ann. of Math. 85 (1967), 430-472.

[71] W. M. Schmidt, Diophantine Approximation. Lecture Notes in Math. 785, Springer, Berlin, 1980.

[72] W. M. Schmidt, Mahler and Koksma classification of points in $\mathbf{R}^{n}$ and $\mathbf{C}^{n}$, Funct. Approx. Comment. Math. 35 (2006), 307-319.

[73] W. M. Schmidt and L. Summerer, Parametric geometry of numbers and applications, Acta Arith. 140 (2009), 67-91.

[74] W. M. Schmidt and L. Summerer, Diophantine approximation and parametric geometry of numbers, Monatsh. Math. 169 (2013), 51-104.

[75] W. M. Schmidt and L. Summerer, Simultaneous approximation to three numbers, Moscow J. Comb. Number Th. 3 (2013), 84-107.

[76] V. G. Sprindžuk, Mahler's problem in metric number theory. Izdat. "Nauka i Tehnika", Minsk, 1967 (in Russian). English translation by B. Volkmann, Translations of Mathematical Monographs, Vol. 25, American Mathematical Society, Providence, R.I., 1969. 
[77] K. I. Tishchenko, On approximation to real numbers by algebraic numbers, Acta Arith. 94 (2000), 1-24.

[78] K. I. Tsishchanka, On approximation of real numbers by algebraic numbers of bounded degree, J. Number Theory 123 (2007), 290-314.

[79] R. C. Vaughan and S. Velani, Diophantine approximation on planar curves: the convergence theory, Invent. Math. 166 (2006), 103-124.

[80] E. Wirsing, Approximation mit algebraischen Zahlen beschränkten Grades, J. reine angew. Math. 206 (1961), 67-77.

[81] D. Zelo, Simultaneous approximation to real and $p$-adic numbers. PhD thesis. Univ. Ottawa. 2009. arXiv:0903.0086.

Yann Bugeaud

Université de Strasbourg

Mathématiques

7, rue René Descartes

67084 STRASBOURG (FRANCE)

bugeaud@math. unistra.fr 